\title{
ON A LOGARITHMIC WEIGHTED POWER DISTRIBUTION: THEORY, MODELLING AND APPLICATIONS
}

\section{CHRISTOPHE CHESNEAU}

Université de Caen

LMNO

Campus II, Sciences 3

14032, Caen

France

e-mail: christophe.chesneau@unicaen.fr

\begin{abstract}
Engineers, economists, hydrologists, social scientists, and behavioural scientists often deal with data belonging to the unit interval. One of the most common approaches for modeling purposes is the use of unit distributions, beginning with the classical power distribution. A simple way to improve its applicability is proposed by the transmuted scheme. We propose an alternative in this article by slightly modifying this scheme with a logarithmic weighted function, thus creating the log-weighted power distribution. It can also be thought of as a variant of the log-Lindley distribution, and some other derived 2020 Mathematics Subject Classification: 60E05, 62E15, 62 F10.
\end{abstract}

Keywords and phrases: power distribution, transmuted power distribution, moments, order statistics, data analysis.

Received July 13, 2021; Revised August 3, 2021

(C) 2021 Scientific Advances Publishers

This work is licensed under the Creative Commons Attribution International License (CC BY 3.0).

http://creativecommons.org/licenses/by/3.0/deed.en_US

Open Access (cc) (1)


unit distributions. We investigate its statistical and functional capabilities, and discuss how it distinguishes between power and transmuted power distributions. Among the functions derived from the log-weighted distribution are the cumulative distribution, probability density, hazard rate, and quantile functions. When appropriate, a shape analysis of them is performed to increase the exibility of the proposed modelling. Various properties are investigated, including stochastic ordering (first order), generalized logarithmic moments, incomplete moments, Rényi entropy, order statistics, reliability measures, and a list of new distributions derived from the main one are offered. Subsequently, the estimation of the model parameters is discussed through the maximum likelihood procedure. Then, the proposed distribution is tested on a few data sets to show in what concrete statistical scenarios it may outperform the transmuted power distribution.

\section{Introduction}

A vast number of continuous distributions with support equal to $(0,1)$, called unit distributions, exist in the statistical literature. Within these unit distributions, some, in particular, have proven useful in a broad range of situations. This is the case for the power (P) distribution described by the following cumulative distribution function (cdf):

$$
F_{P}(x ; \alpha)= \begin{cases}1, & x \geq 1, \\ x^{\alpha}, & x \in(0,1), \\ 0, & x \leq 0,\end{cases}
$$

where $\alpha>0$. Basically, it is the exponentiated version of the uniform distribution over $(0,1)$. More elements, properties and applications of the $\mathrm{P}$ distribution can be found in Johnson et al. [19], Kleiber and Kotz [20], Balakrishnan and Nevzorov [4] and Zaka et al. [36]. Simple extensions of it include the beta distribution studied extensively in Ferrari and CribariNeto [13], Topp-Leone distribution introduced in Topp and Leone [34], Kumaraswami distribution established in Kumaraswamy [23], standard two-sided power distribution proposed in van Dorp and Kotz [35] and transmuted power (TP) distribution developed in Shahzad and Asghar [31]. More sophisticated extensions can be found in the mini review of (Chesneau [7], Table 1), and the references therein. Recent advances in unit distributions may be found in Chesneau [7, 8] and Chesneau et al. [9]. 
The TP distribution, in particular, distinguishes itself from the competition by striking a good balance between simplicity and efficiency. It aims to combine the polynomial functionalities of the transmuted scheme with those of the $\mathrm{P}$ distribution. As a result, the associated cdf is

$$
F_{T P}(x ; \alpha, \lambda)= \begin{cases}1, & x \geq 1, \\ x^{\alpha} w(x ; \alpha, \lambda), & x \in(0,1), \\ 0, & x \leq 0,\end{cases}
$$

where $w(x ; \alpha, \lambda)=1+\lambda\left(1-x^{\alpha}\right)$, with $\lambda \in[-1,1]$ and $\alpha>0$. In some sense, the cdf of the $\mathrm{P}$ distribution is thus multiplied by the weight function $w(x ; \alpha, \lambda)$ for $x \in(0,1)$. The parameter $\lambda$ is a central modulation parameter; the $\mathrm{P}$ distribution is obtained by setting $\lambda=0$, the distribution of the maximum of two independent random variables with the $\mathrm{P}$ distribution is obtained by setting $\lambda=-1$, and the distribution of the minimum of two independent random variables with the $\mathrm{P}$ distribution is obtained by setting $\lambda=1$. All the intermediate decimal values of $\lambda \in[-1,1] /\{-1,0,1\}$ give intermediary distributions of interest. According to Shahzad and Asghar [31], the TP distribution has more flexible functions with interesting non-monotonic curves than the $\mathrm{P}$ distribution. In particular, the probability density function (pdf) has increasing-decreasing shapes and the hazard rate function (hrf) has diverse levels of bathtub shapes, properties that are not observed for the $\mathrm{P}$ distribution. Thanks to its flexible features, the TP distribution can be used quite efficiently to analyze datasets in many fields, such as engineering, economics, hydrology, finance, and behavioural sciences. Further properties of the TP distribution can be found in Shahzad and Asghar [31] and Tanis [33]. 
In this paper, we focus on a simple alternative to the TP distribution. It is based on a cdf similar to the one in Equation (2), but with the following logarithmic weighted function: $w(x ; \alpha, \lambda)=1-\lambda \alpha \log (x)$ with $\lambda \in[0,1]$, for the case $x \in(0,1)$. Again, $\lambda$ is a key modulation parameter; setting $\lambda=0$ yields the $\mathrm{P}$ distribution. All other values of $\lambda$ produce alternative logarithmic distributions. The idea of modulating a logarithmic term in a unit distribution is not new in stricto sensu; we may refer the reader to the log-Lindley distribution established by GómezDéniz et al. [16], as well as the three-parameter power logarithmic distribution by Ahmed et al. [2], and the log-weighted power function distribution by Mandouh and Mohamed [25]. The proposed distribution can be viewed as a simple variant of these modern unit logarithmic distributions, which has not received special attention in the literature, to our knowledge. Its simplicity is a quality; its in-depth study is thus possible on several aspects, which is one aim of the paper.

The following facts motivate the interest in the considered distribution: (i) from a stochastic standpoint, it is strongly linked to the TP proposed and $\mathrm{P}$ distributions, with a clear hierarchy of the corresponding cdfs, (ii) the associated functions, such as the pdf, hrf, and quantile function (qf), are simple and analytically comprehensive, (iii) the asymmetry and tail properties of the pdf and hrf are modulable; in particular, unlike the TP distribution, the pdf has a large panel of decreasing and sharp (mesokurtic) left skewed increasing-decreasing shapes, and the hrf presents flexible bathtub shapes, (iv) the associated moment measures of various kinds, such as generalized logarithmic moments and incomplete moments, are manageable, (v) its randomness can be studied via standard entropy measures, (vi) the associated order statistics and their properties are studyable, with expressible momenttype measures, (vii) its main measure of reliability has a simple structure, (viii) it can be used to define new distributions with diverse numbers of parameters and supports, (ix) from a statistical standpoint, its 
parameters are estimable quite efficiently by the maximum likelihood procedure, and (x) it can outperform the data fitting performance of the TP distribution for some data sets. In the paper, all of these facts are thoroughly explored.

The outline of the paper is as follows: Section 2 details the proposed log-weighted power distribution with an emphasis on the key functions. We discuss its quantile properties in Section 3. Section 4 performs a moment analysis, and Section 5 an entropy analysis. The order statistics of the proposed distribution are developed in Section 6. A measure of reliability is given in Section 7. Some distributional results are presented in Section 8. Applications in statistics are given in Section 9. Concluding remarks are formulated in Section 10.

\section{The Log-Weighted Power Distribution}

This section presents the proposed alternative to the transmuted power distribution, along with some of its functional facets.

\subsection{Description}

The following result shows a mathematically valid cdf, which will be central to the investigation.

Proposition 2.1. Let $\alpha>0$ and $\lambda \in[0,1]$. Then, the following function has the properties of a valid cdf:

$$
F(x)= \begin{cases}1, & x \geq 1, \\ x^{\alpha}[1-\lambda \alpha \log (x)], & x \in(0,1), \\ 0, & x \leq 0 .\end{cases}
$$


Proof. It is clear that $\lim _{x \rightarrow 0} F(x)=0$ and $\lim _{x \rightarrow 1} F(x)=1$. Moreover, $F(x)$ is continuous for $x \in \mathbb{R}$. For $x \in(0,1)$, its derivative is obtained as $F^{\prime}(x)=\alpha x^{\alpha-1}[1-\lambda-\lambda \alpha \log (x)]$. Since $1-\lambda>0$ and $-\lambda \alpha \log (x)>0$, we have $F^{\prime}(x)>0$, implying that $F(x)$ is strictly increasing for $x \in(0,1)$. All these facts combined validate $F(x)$ as a cdf.

To our knowledge, the two-parameter cdf presented in Proposition 2.1 has not received special attention in the literature, but we can not claim that it is completely new; it has some connections with existing functions. First, it coincides with the cdf of the log-Lindley distribution under the following specific configuration: $\lambda=1 /\left(1+\lambda_{*} \alpha\right)$, where $\lambda_{*}$ denotes the " $\lambda$ " in the former definition of the log-Lindley distribution (see GómezDéniz et al. [16]). Note that this particular $\lambda$ is connected with $\alpha$ and can not be equal to 0 . In addition, such a reparameterization could affect the numerical estimation of the parameters. On these points, the proposed cdf differs. Also, the proposed distribution is a special case of the threeparameter power logarithmic distribution by Ahmed et al. [2]; it appears by taking " $\alpha_{*}=\alpha$ ", " $\beta_{*}=1-\lambda$ ", and " $\delta_{*}=\lambda \alpha$ ", where $\alpha_{*}, \beta_{*}$ and $\delta_{*}$ refer to the parameters of the three-parameter power logarithmic distribution. However, the simplicity in the definition of the proposed distribution is a quality, which allows an in-depth study for both the mathematical and practical aspects. For this reason, we will take a close look at it in this article. On the other hand, in the case of $\lambda=1$, it corresponds to the so-called lookback distortion, which is sometimes used to define distortion risk measures in finance and insurance applications (see Denuit et al. [12]). 
Hence, we present the log-weighted power (LP) distribution, specified by the following cdf:

$$
F_{\mathrm{LP}}(x ; \alpha, \lambda)= \begin{cases}1, & x \geq 1, \\ x^{\alpha}[1-\lambda \alpha \log (x)], & x \in(0,1), \\ 0, & x \leq 0,\end{cases}
$$

with $\lambda \in[0,1]$ and $\alpha>0$. It is also denoted as $\operatorname{LP}(\alpha, \lambda)$ distribution to specify the parameters. As a first remark, it generalizes the $\mathrm{P}$ distribution; the cdf of the $\mathrm{P}$ distribution is obtained by taking $\lambda=0$ in Equation (3), and it provides a real alternative to the TP distribution. This last claim is illustrated in the next result.

Proposition 2.2. The following inequalities hold: For any $\lambda \in[0,1]$ and $x \in \mathbb{R}$, we have

$$
F_{\mathrm{P}}(x ; \alpha) \leq F_{\mathrm{TP}}(x ; \alpha, \lambda) \leq F_{\mathrm{LP}}(x ; \alpha, \lambda) .
$$

Proof. For $x \leq 0$ or $x \geq 1$, the cdfs are equal to the same constants, 0 or 1 . For $\lambda=0$ and $x \in(0,1)$, they are equal to $x^{\alpha}$. For $\lambda>0$ and $x \in(0,1)$, since $1-x^{\alpha}>0$ and $\lambda>0$, we have $F_{\mathrm{P}}(x ; \alpha)=x^{\alpha}<x^{\alpha}$ $\left[1+\lambda\left(1-x^{\alpha}\right)\right]=F_{\mathrm{TP}}(x ; \alpha, \lambda)$. The first inequality is established. For $y \in(0,1)$, the following inequality is valid: $\log (y)<y-1$. By taking $y=x^{\alpha} \in(0,1)$, we obtain $F_{\mathrm{LP}}(x ; \alpha, \lambda)=x^{\alpha}\left[1-\lambda \log \left(x^{\alpha}\right)\right]>x^{\alpha}[1+\lambda$ $\left.\left(1-x^{\alpha}\right)\right]=F_{\mathrm{TP}}(x ; \alpha, \lambda)$. The second inequality is obtained, ending the proof of the proposition.

The result above means that, for $\lambda \in[0,1]$, the TP distribution first order stochastically dominates the LP distribution, implying different objectives in terms of data fitting, among other things. More differences between the TP and LP distributions will be discussed later. 
Figure 1 presents a panel of shapes of the cdf of the LP distribution.

$F_{L P}(x ; \alpha, 0)$

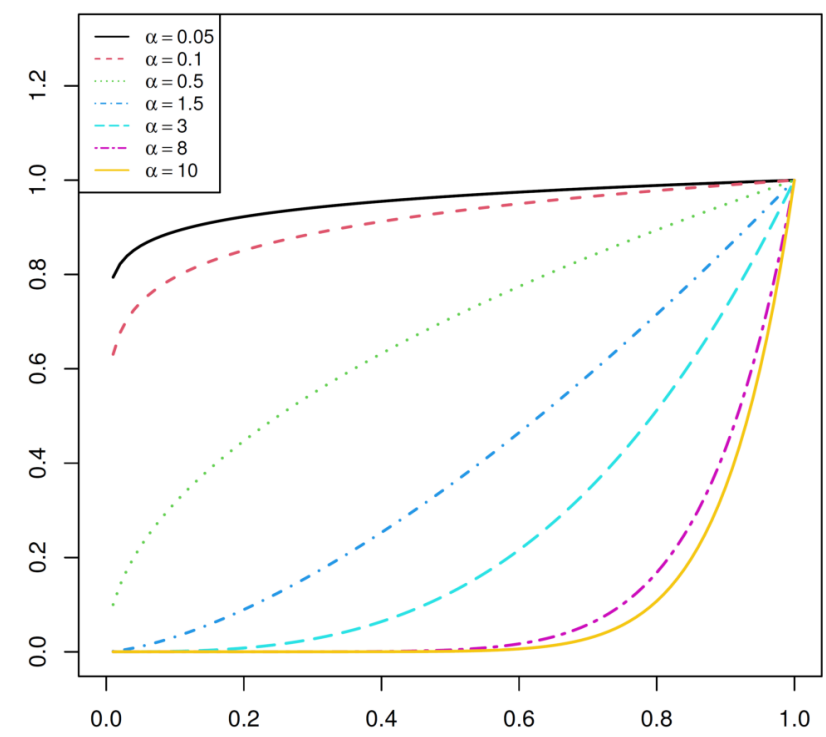

$F_{L P}(x ; \alpha, 0.3)$

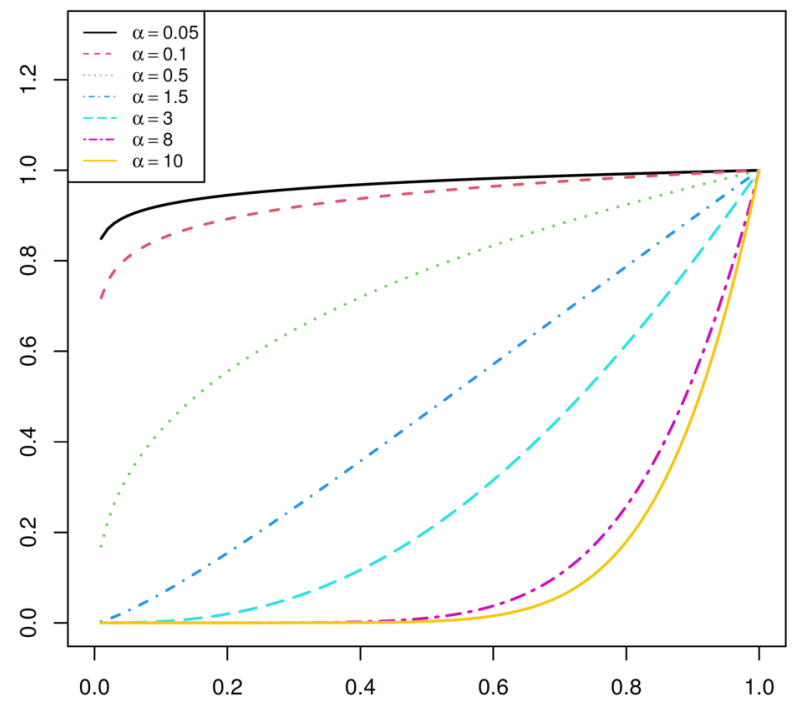


$F_{L P}(x ; \alpha, 0.6)$

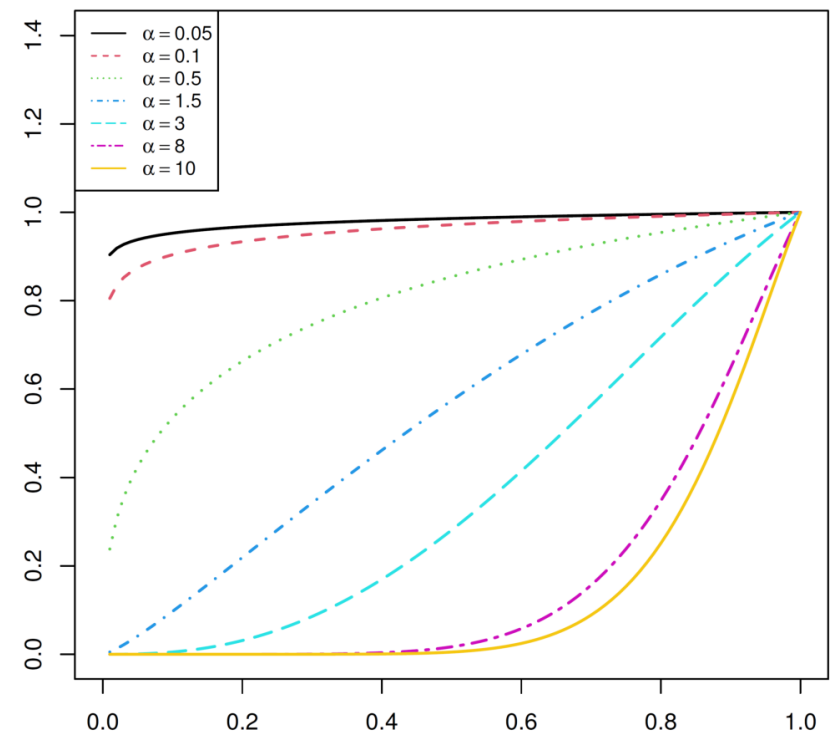

$\mathrm{F}_{\mathrm{LP}}(\mathrm{x} ; \alpha, 1)$

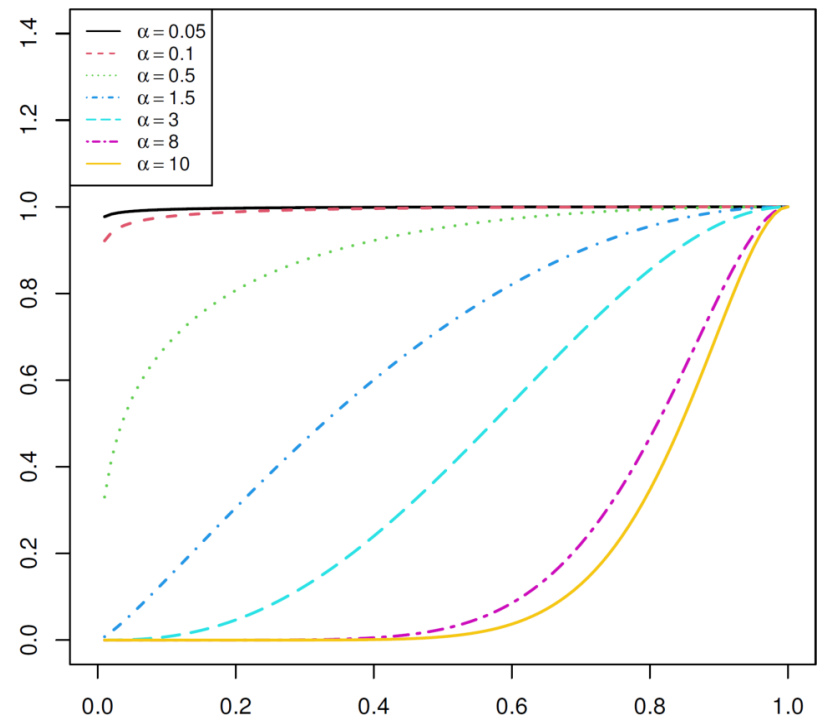

Figure 1. Curves for the $\operatorname{cdf} F_{\mathrm{LP}}(x ; \alpha, \lambda)$ for various values of $\alpha$ and $\lambda$. 
Various concave and convex shapes, including diagonal and angular shapes, attest to the flexibility of the cdf.

\subsection{Distributional functions}

We are interested in the expressions of the main functions of the LP distribution. First, the pdf is derived from Equation (3) upon differentiation, and is given implicitly in the proof of Proposition 2.1. It is expressed as

$$
f_{\mathrm{LP}}(x ; \alpha, \lambda)=\left(\begin{array}{ll}
\alpha x^{\alpha-1}[1-\lambda-\lambda \alpha \log (x)], & x \in(0,1), \\
0, & x \notin(0,1) .
\end{array}\right.
$$

For $x \in(0,1)$, it is also related to $F_{\mathrm{LP}}(x ; \alpha, \lambda)$ as

$$
\begin{aligned}
f_{\mathrm{LP}}(x ; \alpha, \lambda) & =\frac{\alpha}{x} F_{\mathrm{LP}}(x ; \alpha, \lambda)-\alpha \lambda x^{\alpha-1} \Leftrightarrow F_{\mathrm{LP}}(x ; \alpha, \lambda) \\
& =\frac{x}{\alpha} f_{\mathrm{LP}}(x ; \alpha, \lambda)+\lambda x^{\alpha} .
\end{aligned}
$$

This relationship will have its utility in some mathematical developments.

Let us examine this pdf analytically using standard tools. For $x \in(0,1)$, the following limits hold:

$$
\lim _{x \rightarrow 0} f_{\mathrm{LP}}(x ; \alpha, \lambda)=\left\{\begin{array}{lc}
0, & \alpha>1, \\
+\infty, & \alpha \in(0,1],
\end{array} \quad \lim _{x \rightarrow 1} f_{\mathrm{LP}}(x ; \alpha, \lambda)=\alpha(1-\lambda) .\right.
$$

The shape behaviour of the pdf is studied below.

Proposition 2.3. For $\alpha \in(0,1]$ and $\lambda \in[0.1], f_{\mathrm{LP}}(x ; \alpha, \lambda)$ is strictly decreasing for $x \in(0,1)$. 
- For $\alpha>1$ and $\lambda \in((\alpha-1) /(2 \alpha-1), 1], f_{\mathrm{LP}}(x ; \alpha, \lambda)$ is increasingdecreasing for $x \in(0,1)$, and the maximal point is given as

$$
x_{*}=e^{-[\alpha(2 \lambda-1)+1-\lambda] /[(\alpha-1) \alpha \lambda]} .
$$

- For $\alpha>1$ and $\lambda \in[0,(\alpha-1) /(2 \alpha-1)], f_{\mathrm{LP}}(x ; \alpha, \lambda)$ is strictly increasing for $x \in(0,1)$.

Proof. As a special case, for $\alpha=1$ and $x \in(0,1), f_{L P}(x ; \alpha, \lambda)=1-\lambda$ $-\lambda \log (x)$ which is clearly decreasing. It is assumed in the following that $\alpha \neq 1$. The derivative of $f_{\mathrm{LP}}(x ; \alpha, \lambda)$ is given as

$$
f_{\mathrm{LP}}^{\prime}(x ; \alpha, \lambda)=-\alpha x^{\alpha-2}[(\alpha-1) \alpha \lambda \log (x)+\alpha(2 \lambda-1)+1-\lambda] .
$$

Therefore $f_{\mathrm{LP}}(x ; \alpha, \lambda)$ is strictly decreasing if and only if $f_{\mathrm{LP}}^{\prime}(x ; \alpha, \lambda)<0$. This inequality is equivalent to $(\alpha-1) \alpha \lambda \log (x)+\alpha(2 \lambda-1)+1-\lambda>0$. Since $\lim _{x \rightarrow 0} \log (x)=-\infty$ and $\log (1)=0$, this inequality is possible for all $x \in(0,1)$ if and only if $\alpha \in(0,1)$ and $\alpha(2 \lambda-1)+1-\lambda>0$, so $\lambda(2 \alpha-1)+1-\alpha>0$.

- If $\alpha \in[1 / 2,1)$, the inequality is obviously satisfied for all $\lambda \in[0,1]$.

- If $\alpha \in(0,1 / 2)$, this inequality becomes $\lambda<(\alpha-1) /(2 \alpha-1)$, and this upper bound is strictly superior to 1 , so we can keep the initial assumption on $\lambda$ without restriction, so $\lambda \in[0,1]$.

As a result, for $\alpha \in(0,1]$ and $\lambda \in[0,1], f_{\mathrm{LP}}(x, \alpha, \lambda)$ is strictly decreasing for $x \in(0,1)$.

- Based on Equation (5), we have $f_{\mathrm{LP}}^{\prime}\left(x_{*} ; \alpha, \lambda\right)=0$ if and only if $x_{*}=e^{-[\alpha(2 \lambda-1)+1-\lambda] /[(\alpha-1) \alpha \lambda]}$. Consequently, $f_{\mathrm{LP}}^{\prime}(x ; \alpha, \lambda)$ is non-monotonic if $x_{*} \in(0,1)$. This condition is equivalent to $[\alpha(2 \lambda-1)+1-\lambda] /[(\alpha-1) \alpha \lambda]>0$ and $\lambda>0$. 
- If $\alpha>1$, this inequality is equivalent to $\alpha(2 \lambda-1)+1-\lambda>0$, hence $\lambda(2 \alpha-1)+1-\alpha>0$, so $\lambda>(\alpha-1) /(2 \alpha-1)$, and this lower bound belongs to $(0,1)$.

- If $\alpha<1$, this inequality becomes $\alpha(2 \lambda-1)+1-\lambda<0$, so $\lambda(2 \alpha-1)+$ $1-\alpha<0$. Therefore, if $\alpha \in[1 / 2,1)$, this inequality can not be satisfied because the left term is positive. If $\alpha \in(0,1 / 2)$, we have $\lambda>(\alpha-1) /$ $(2 \alpha-1)$, and this lower bound is strictly superior to 1 . This contradicts the initial assumption that $\lambda \leq 1$.

As a result, for $\alpha>1$ and $\lambda \in((\alpha-1) /(2 \alpha-1), 1], f_{\mathrm{LP}}(x ; \alpha, \lambda)$ is non-monotonic for $x \in(0,1)$ with an extremum at the point $x=x_{*}=$ $e^{-[\alpha(2 \lambda-1)+1-\lambda] /[(\alpha-1) \alpha \lambda]}$. Since $\lim _{x \rightarrow 0} f_{\mathrm{LP}}(x ; \alpha, \lambda)=0$ with $f_{\mathrm{LP}}(x ; \alpha, \lambda)$ $>0$ for all $x \in(0,1)$, this point is a maximum point and the function is increasing-decreasing.

- The last claim is a consequence of the two items above. Indeed, we have shown that, if $f_{\mathrm{LP}}(x ; \alpha, \lambda)$ is non-monotonic, it has only one mode. So it is monotonic in other cases. And since the decreasing case is wellidentified, we deduce the increasing case as the last possible case, reached with $\alpha>1$ and $\lambda \in[0,(\alpha-1) /(2 \alpha-1)]$. As an alternative proof of this claim, we can proceed analytically as the first item to obtain the same result.

This ends the proof of the proposition.

As a consequence of Proposition 2.3, the LP distribution is unimodal for the case $\alpha>1$ and $\lambda \in((\alpha-1) /(2 \alpha-1), 1]$, and the mode is given by $x_{*}$. At this value, we have the maximum of the pdf given as

$$
f_{\max }=f_{\mathrm{LP}}\left(x_{*} ; \alpha, \lambda\right)=\frac{\alpha^{2} \lambda}{\alpha-1} e^{-[\alpha(2 \lambda-1)+1-\lambda] /(\alpha \lambda)} .
$$

We thus see the impact of $\lambda$ and $\alpha$ in this maximum. Figure 2 illustrates the shape behaviour of the pdf of the LP distribution. 
ON A LOGARITHMIC WEIGHTED POWER ...

$f_{L P}(x ; \alpha, 0)$

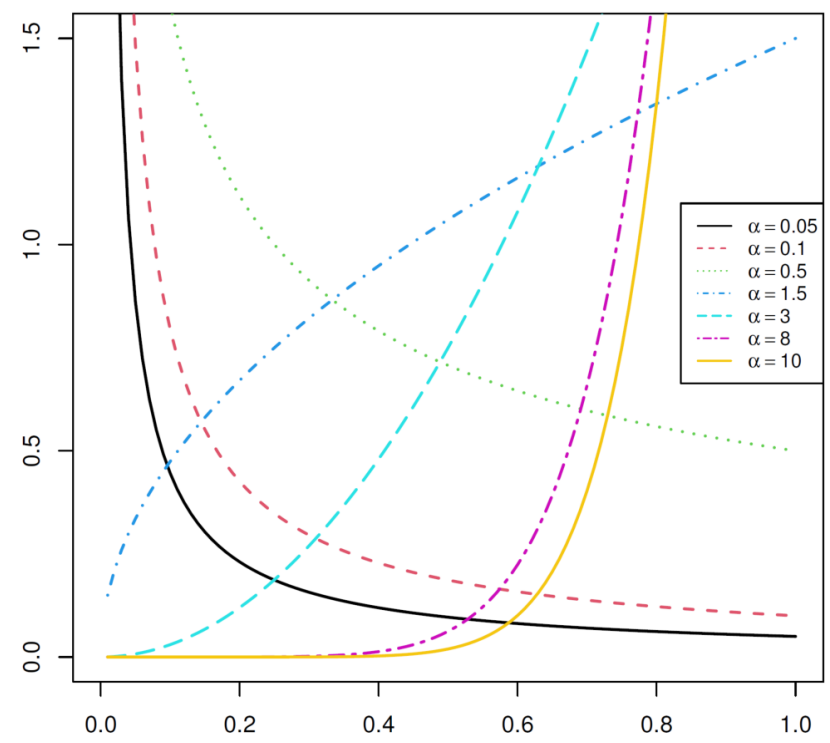

$\mathrm{f}_{\mathrm{LP}}(\mathrm{x} ; \alpha, 0.3)$

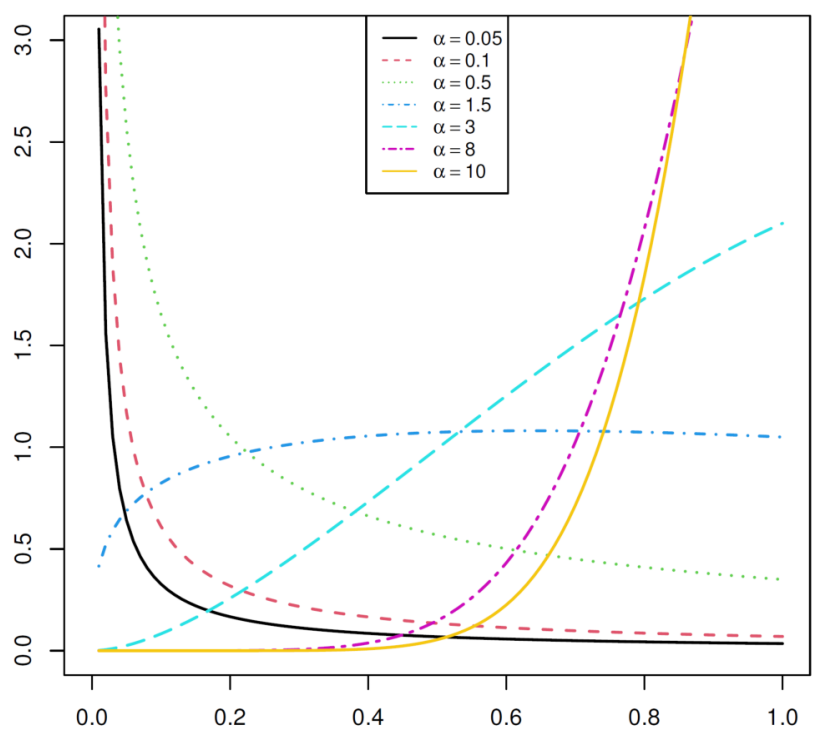



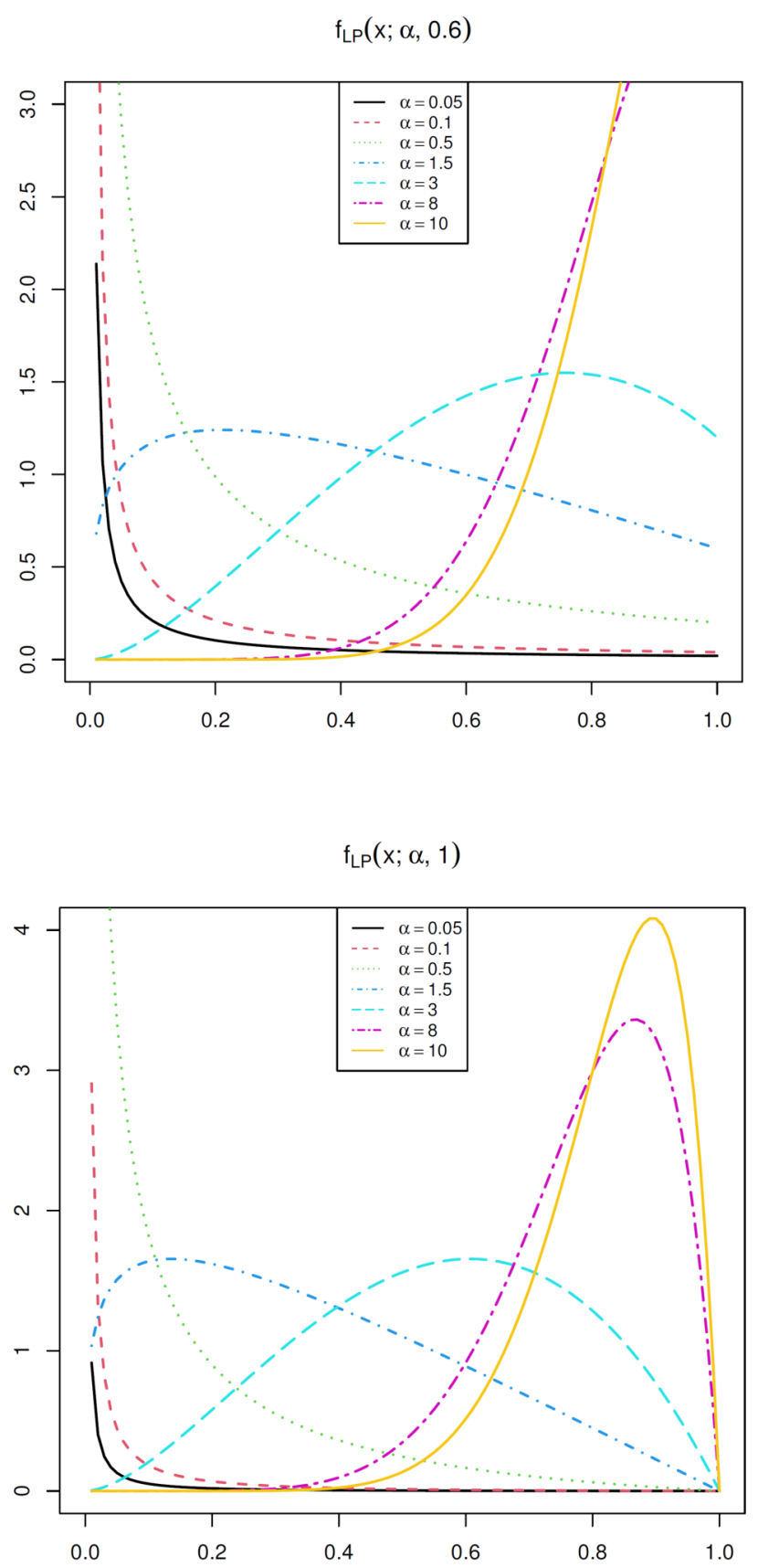

Figure 2. Curves for the pdf $f_{\mathrm{LP}}(x ; \alpha, \lambda)$ for various values of $\alpha$ and $\lambda$. 
From Figure 2, it can be noted that, unlike the pdf of the TP distribution, the pdf of the LP distribution has a large panel of decreasing and sharp (mesokurtic) left skewed increasing-decreasing shapes.

The hrf is calculated as

$$
\begin{aligned}
h_{\mathrm{LP}}(x ; \alpha, \lambda) & =\frac{f_{\mathrm{LP}}(x ; \alpha, \lambda)}{1-F_{\mathrm{LP}}(x ; \alpha, \lambda)} \\
& = \begin{cases}\frac{\alpha x^{\alpha-1}[1-\lambda-\lambda \alpha \log (x)]}{1-x^{\alpha}[1-\lambda \alpha \log (x)]}, & x \in(0,1), \\
0, & x \notin(0,1) .\end{cases}
\end{aligned}
$$

Let us investigate this hrf by using standard analytical tools. For $x \in(0,1)$, the following limits hold:

$$
\begin{aligned}
\lim _{x \rightarrow 0} h_{\mathrm{LP}}(x ; \alpha, \lambda) & =\left\{\begin{array}{cc}
0, & \alpha>1, \\
+\infty, & \alpha \in(0,1],
\end{array} \quad \lim _{x \rightarrow 1} h_{\mathrm{LP}}(x ; \alpha, \lambda)\right. \\
& =\lim _{x \rightarrow 1} \frac{1}{1-x}=+\infty .
\end{aligned}
$$

Therefore, for all the values of the parameters, the hrf "explodes" at the neighbourhood of $x=1$, and this with a harmonic decrease.

The following result shows that the hrf can be an increasing function, depending on the values of $\alpha$ and $\lambda$.

Proposition 2.4. If $\alpha>5 / 4$ and $\lambda>0$, then $h_{\mathrm{LP}}(x ; \alpha, \lambda)$ is increasing.

Proof. The proof is based on a result established by Glaser [15]. In the context of the LP distribution, it may be formulated as follows. Let us set

$$
\psi(x ; \alpha, \lambda)=-\frac{f_{\mathrm{LP}}^{\prime}(x ; \alpha, \lambda)}{f_{\mathrm{LP}}(x ; \alpha, \lambda)}
$$


Then, according to Glaser [15], if $\psi^{\prime}(x ; \alpha, \lambda)$ is strictly positive for $x \in(0,1)$, then $h_{\mathrm{LP}}(x ; \alpha, \lambda)$ is increasing. Here, after some developments and simplifications, we have

$$
\psi(x ; \alpha, \lambda)=\frac{(\alpha-1) \alpha \lambda \log (x)+\alpha(2 \lambda-1)+1-\lambda}{x[1-\lambda-\lambda \alpha \log (x)]},
$$

and

$$
\psi^{\prime}(x ; \alpha, \lambda)=\frac{(\alpha-1) \alpha^{2} \lambda^{2}[\log (x)]^{2}+\alpha \lambda(\alpha(3 \lambda-2)-2 \lambda+2) \log (x)}{+\alpha^{2} \lambda^{2}+\alpha\left(2 \lambda^{2}-3 \lambda+1\right)-(1-\lambda)^{2}} .
$$

Hence, for determining the sign of $\psi^{\prime}(x ; \alpha, \lambda)$, it is enough to find the sign of the numerator term. This term is of the form $P_{\alpha}(\log (x))$, where $P_{\alpha}(y)=a_{\alpha} y^{2}+b_{\alpha} y+c_{\alpha}$, where $a_{\alpha}=(\alpha-1) \alpha^{2} \lambda^{2}, b_{\alpha}=\alpha \lambda(\alpha(3 \lambda-2)-2$ $\lambda+2)$ and $c_{\alpha}=\alpha^{2} \lambda^{2}+\alpha\left(2 \lambda^{2}-3 \lambda+1\right)-(1-\lambda)^{2}$. After a fastidious development, the discriminant of $P_{\alpha}(y)$ is given as $\Delta=b_{\alpha}^{2}-4 a_{\alpha} c_{\alpha}=$ $(5-4 \alpha) \alpha^{4} \lambda^{4}$. Therefore, we have $\Delta<0$ for $\alpha>5 / 4$ and $\lambda>0$, implying that $P_{\alpha}(y)$ has a constant sign for all $y \in \mathbb{R}$. Hence, let us study the sign of the simple case described by $P_{\alpha}(1)=c_{\alpha}=\left(\alpha^{2}+2 \alpha-1\right)$ $\lambda^{2}+(2-3 \alpha) \lambda+\alpha-1$. This quantity can be thought of as a polynomial in terms of $\lambda$, which we denote as $Q_{\alpha}(\lambda)$. It has the discriminant $\Delta_{*}=5 \alpha^{2}-4 \alpha^{3}$, which is strictly negative if $\alpha>5 / 4$. Therefore, the sign of $Q_{\alpha}(\lambda)$ is constant, and it is in particular the one of $Q_{\alpha}(1)=\alpha^{2}>0$. Therefore, $P_{\alpha}(\log (x))>0$, implying that $w^{\prime}(x ; \alpha, \lambda)>0$ and, by Glaser [15], $h_{\mathrm{LP}}(x ; \alpha, \lambda)$ is increasing. The proposition is demonstrated.

For the case of $\alpha \in(0,1]$, in view of the limit properties of $h_{\mathrm{LP}}(x ; \alpha, \lambda)$, it is clear that $h_{\mathrm{LP}}(x ; \alpha, \lambda)$ has at least a minimum point. Due to a high level of complexity in terms of analytical developments, we propose a graphical analysis to complete these results. We plot several curves of this hrf in Figure 3. 
ON A LOGARITHMIC WEIGHTED POWER ...

$\mathrm{h}_{\mathrm{LP}}(\mathrm{x} ; \alpha, 0)$

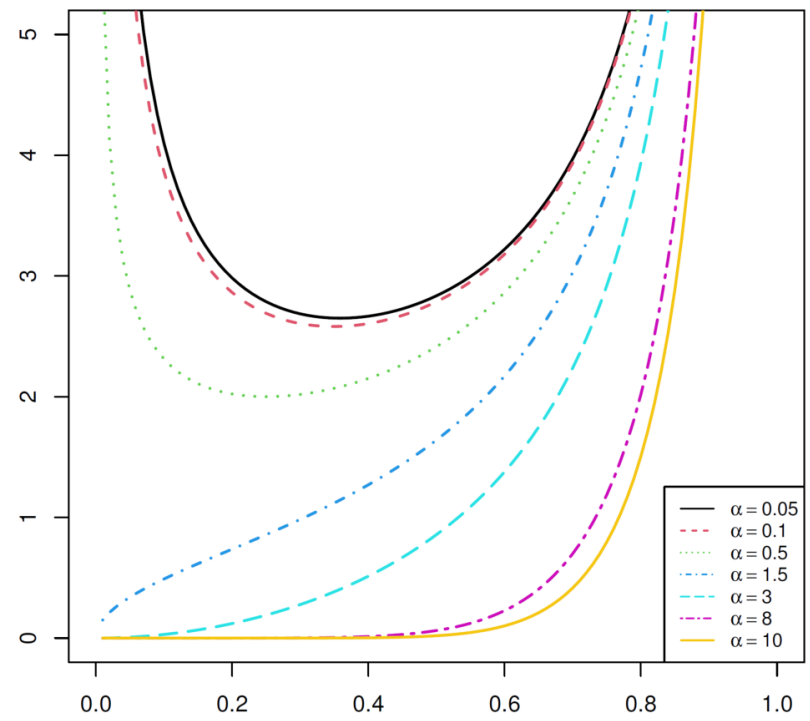

$h_{L P}(x ; \alpha, 0.3)$

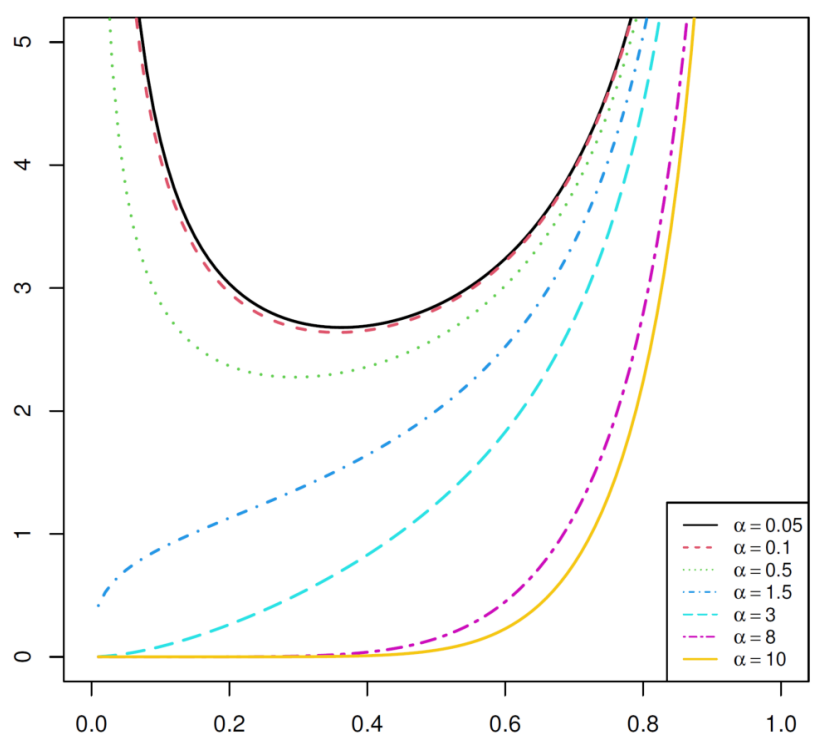


$h_{L P}(x ; \alpha, 0.6)$

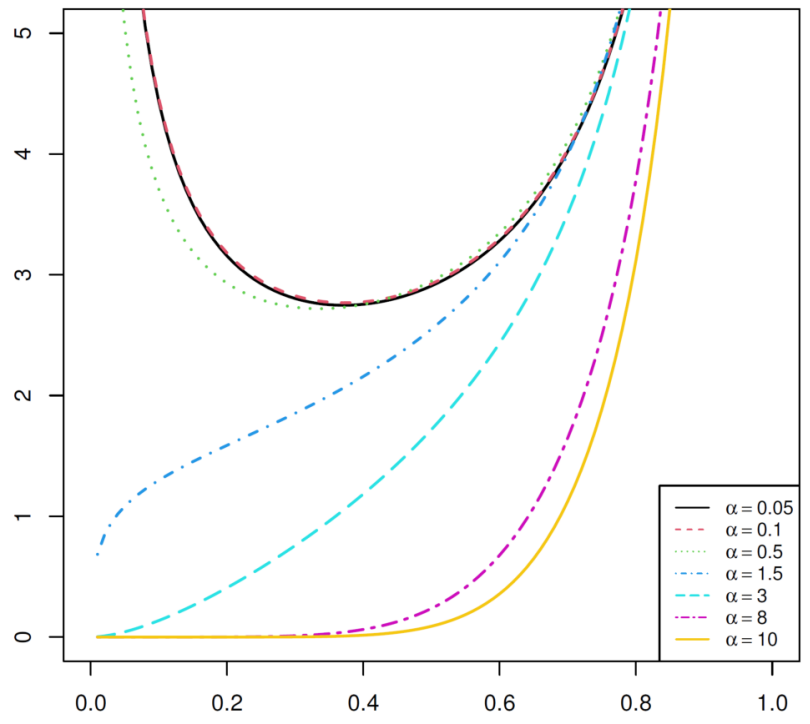

$\mathrm{h}_{\mathrm{LP}}(\mathrm{x} ; \alpha, 1)$

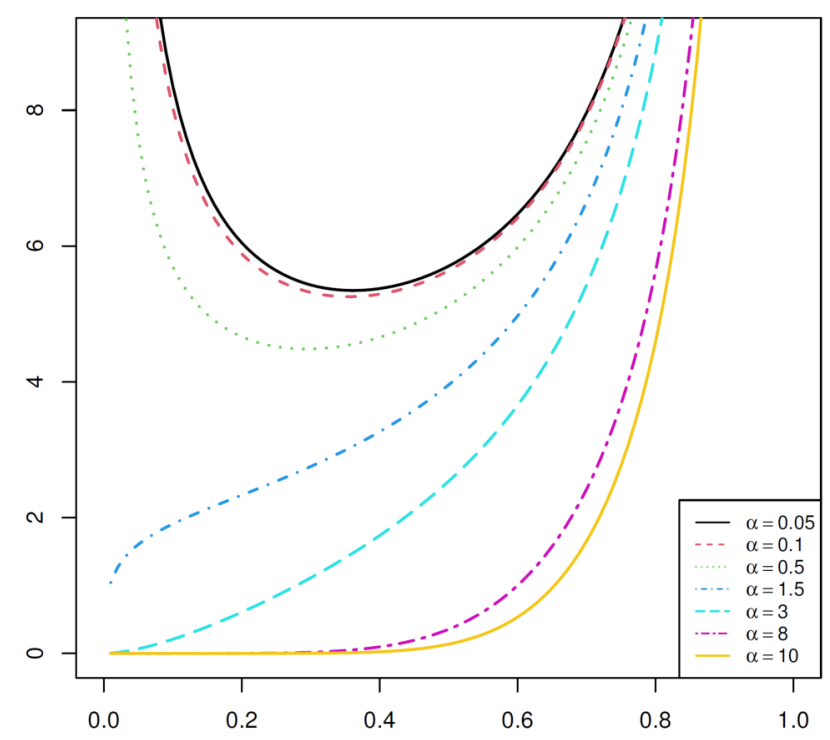

Figure 3. Curves for the hrf $h_{\mathrm{LP}}(x ; \alpha, \lambda)$ for various values of $\alpha$ and $\lambda$. 
From Figure 3 , we see that $h_{\mathrm{LP}}(x ; \alpha, \lambda)$ has increasing and bathtub shapes, which is consistent with Proposition 2.4 and the formulated remarks. The wide range of bathtub shapes of the hrf of the LP distribution is not observed for the hrf of the TP distribution.

\section{Quantile Analysis}

Quantiles are cut points in a probability distribution that split its range into continuous intervals with equal probability. They are specific values of the qf. The qf of the LP distribution is determined in the next result.

Theorem 3.1. The of of the LP distribution is expressed as

$$
Q_{\mathrm{LP}}(y ; \alpha, \lambda)=\left\{\begin{array}{ll}
y^{1 / \alpha}, & \lambda=0, \\
y^{1 / \alpha}\left[-\lambda W_{-1}\left(-\frac{y}{\lambda} e^{-1 / \lambda}\right)\right]^{-1 / \alpha}, & \lambda>0,
\end{array} \quad y \in(0,1),\right.
$$

where $W_{-1}(x)$ is the secondary real branch of the Lambert function.

Proof. We have $Q_{\mathrm{LP}}(y ; \alpha, \lambda)=F_{\mathrm{LP}}^{-1}(y ; \alpha, \lambda)$. Thus, it satisfies the following functional equation: $F_{\mathrm{LP}}(x ; \alpha, \lambda)=y$ according to $x$. The case $\lambda=0$ is immediate; we have $y=x^{\alpha}$ implying the desired result. For the case $\lambda>0$, with step-by-step progression, we get

$$
\begin{aligned}
y= & F_{L P}(x ; \alpha, \lambda) \quad \Leftrightarrow \quad y=x^{\alpha}[1-\lambda \alpha \log (x)] \\
& \Leftrightarrow \quad \log \left(x^{-\alpha}\right)=\frac{y}{\lambda} x^{-\alpha}-\frac{1}{\lambda} \quad \Leftrightarrow \quad x^{-\alpha}=e^{y x^{-\alpha} / \lambda} e^{-1 / \lambda} \\
& \Leftrightarrow \quad-\frac{y}{\lambda} x^{-\alpha} e^{-y x^{-\alpha} / \lambda}=-\frac{y}{\lambda} e^{-1 / \lambda} \Leftrightarrow-\frac{y}{\lambda} x^{-\alpha}=W_{-1}\left(-\frac{y}{\lambda} e^{-1 / \lambda}\right) \\
& \Leftrightarrow \quad x=y^{1 / \alpha}\left[-\lambda W_{-1}\left(-\frac{y}{\lambda} e^{-1 / \lambda}\right)\right]^{-1 / \alpha} .
\end{aligned}
$$

The proof ends. 
Figure 4 illustrates the shape behaviour of the of of the LP distribution.

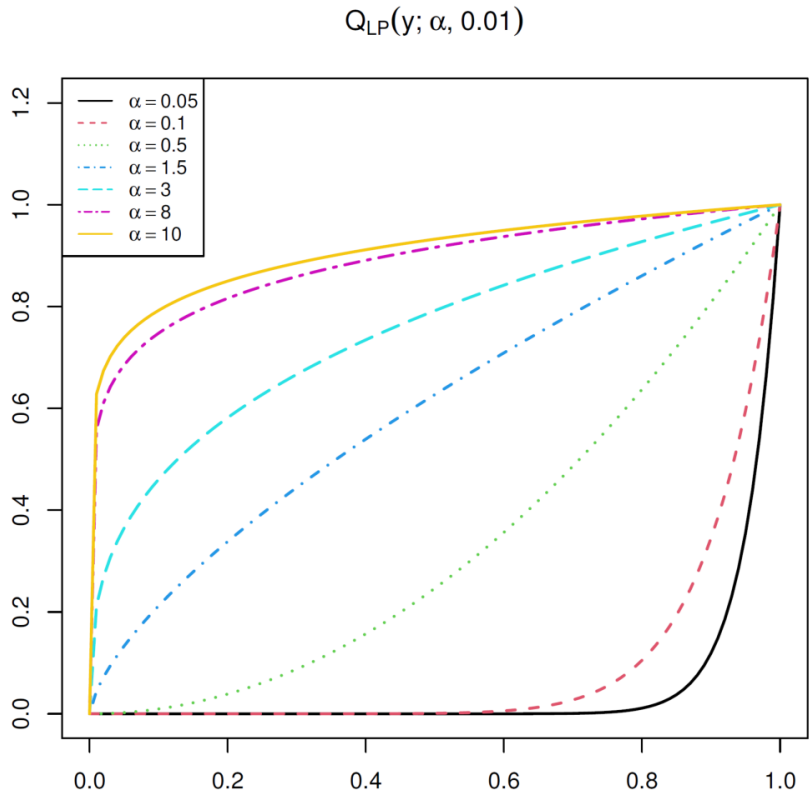

$Q_{L P}(y ; \alpha, 0.3)$

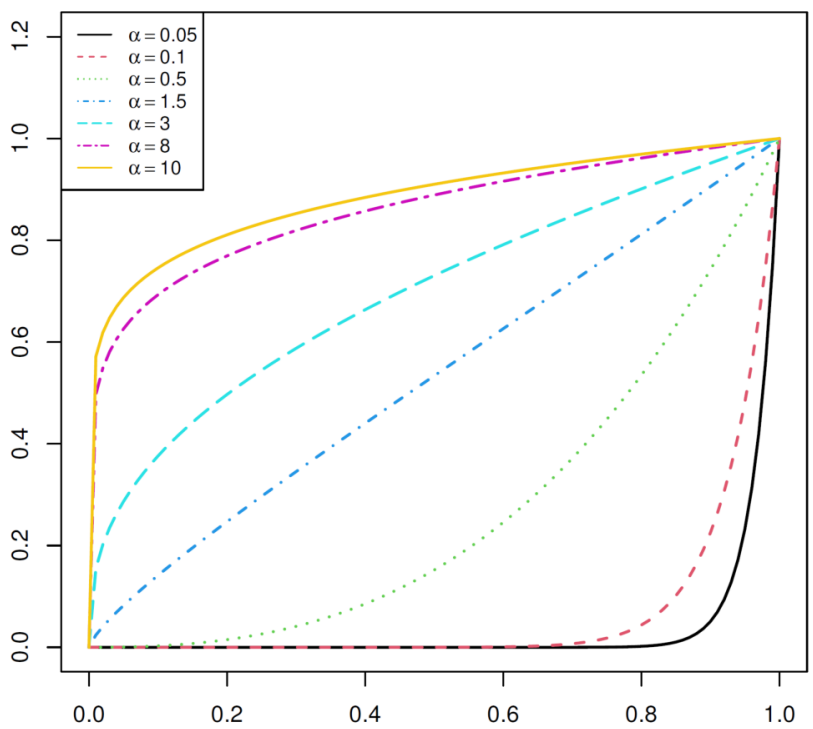


$\mathrm{Q}_{\mathrm{LP}}(\mathrm{y} ; \alpha, 0.6)$

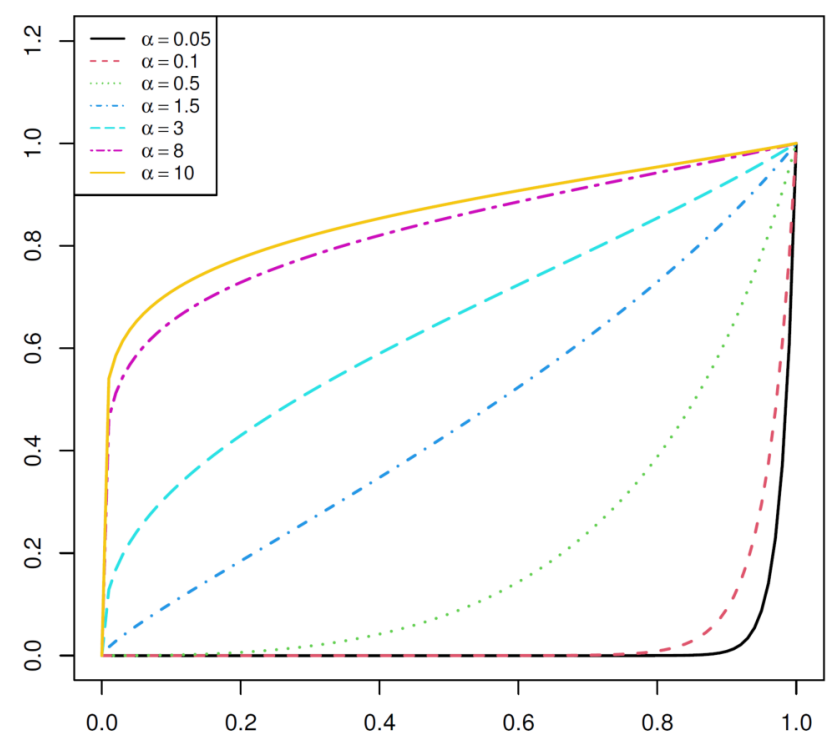

$Q_{L P}(y ; \alpha, 1)$

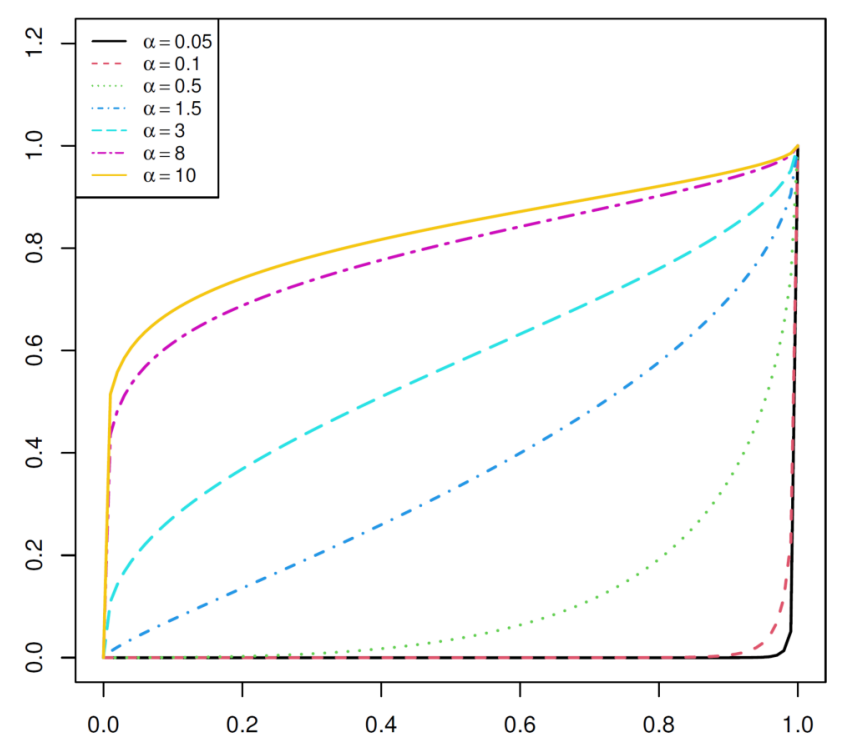

Figure 4. Curves for the of $Q_{\mathrm{LP}}(y ; \alpha, \lambda)$ for various values of $\alpha$ and $\lambda$. 
The flexibility of the of is demonstrated by a variety of concave and convex shapes, including diagonal and angular shapes.

Based on the qf, we can determine the median of the LP distribution defined by $M_{\mathrm{LP}}=Q_{\mathrm{LP}}(1 / 2 ; \alpha, \lambda)$, corresponding to

$$
M_{\mathrm{LP}}= \begin{cases}2^{-1 / \alpha}, & \lambda=0, \\ 2^{-1 / \alpha}\left[-\lambda W_{-1}\left(-\frac{1}{2 \lambda} e^{-1 / \lambda}\right)\right]^{-1 / \alpha}, & \lambda>0 .\end{cases}
$$

The two other quartiles can be expressed in a similar manner, by taking the values $u=1 / 4$ and $u=3 / 4$ in the definition of $Q_{\mathrm{LP}}(u ; \alpha, \lambda)$.

Also, the following result in distribution holds. Let $U$ be a random variable with the uniform distribution on $(0,1)$ and $V=Q_{\mathrm{LP}}(U ; \alpha, \lambda)$, corresponding to

$$
V= \begin{cases}U^{1 / \alpha}, & \lambda=0, \\ U^{1 / \alpha}\left[-\lambda W_{-1}\left(-\frac{U}{\lambda} e^{-1 / \lambda}\right)\right]^{-1 / \alpha}, & \lambda>0 .\end{cases}
$$

Then $V$ follows the $\operatorname{LP}(\alpha, \lambda)$ distribution. This result allows the generation of values from the LP distribution based on generated values from the uniform distribution on $(0,1)$. This can serve as the basis of a simulation study to evaluate the efficiency of different estimates of the parameters of the LP distribution.

More generally, the analytical expression of the of allows for a more detailed quantile study of the LP distribution, including expressions for the quantile density and hazard qfs, and diverse asymmetry and plateness quantile measures. The role of these quantities in survival experiments is shown in Gilchrist [14] and Nair and Sankaran [26], among others. Also, the construction of the quantile LP regression model has become possible. 


\section{Moments Analysis}

\subsection{Classical approach}

Various types of moments of a real random variable provide indicators of important characteristics of this variable. These indicators can be categorized as central, dispersed, or shape. Here, we first begin by expressing the generalized logarithmic moments of the LP distribution.

Theorem 4.1. Let $r$ be an integer such that $r>-\alpha, s$ be a positive integer, and $X$ be a random variable with the LP distribution. Then, the $(r, s)$-th generalized logarithmic moment of $X$ is given as

$$
m_{r, s}^{\log }=\mathbb{E}\left(X^{r}[\log (X)]^{s}\right)=\frac{\alpha(-1)^{s} s !}{(r+\alpha)^{s+2}}[r(1-\lambda)+\alpha(1+\lambda s)] .
$$

Proof. We have

$$
\begin{aligned}
m_{r, s}^{\log } & =\int_{-\infty}^{+\infty} x^{r}[\log (x)]^{s} f_{\mathrm{LP}}(x ; \alpha, \lambda) d x \\
& =\alpha \int_{0}^{1} x^{r+\alpha-1}[\log (x)]^{s}[1-\lambda-\lambda \alpha \log (x)] d x \\
& =\alpha\left[(1-\lambda) \int_{0}^{1} x^{r+\alpha-1}[\log (x)]^{s} d x-\lambda \alpha \int_{0}^{1} x^{r+\alpha-1}[\log (x)]^{s+1} d x\right]
\end{aligned}
$$

According to (Gradshteyn and Ryzhik [17], Equation (4.2726)), the following formula is valid for any real number $\mu>-1$ :

$$
\int_{0}^{1} x^{\mu}[\log (x)]^{s} d x=(-1)^{s} \frac{s !}{(\mu+1)^{s+1}}
$$


Therefore

$$
\begin{aligned}
m_{r, s}^{\log } & =\alpha\left[(1-\lambda)(-1)^{s} \frac{s !}{(r+\alpha)^{s+1}}-\lambda \alpha(-1)^{s+1} \frac{(s+1) !}{(r+\alpha)^{s+2}}\right] \\
& =\frac{\alpha(-1)^{s} s !}{(r+\alpha)^{s+1}}\left[1-\lambda+\lambda \alpha \frac{s+1}{r+\alpha}\right]=\frac{\alpha(-1)^{s} s !}{(r+\alpha)^{s+2}}[r(1-\lambda)+\alpha(1+\lambda s)] .
\end{aligned}
$$

The desired result is obtained.

From Theorem 4.1, we immediately derive the following momentstype measures:

- For any positive integer $r$, the $r$-th ordinary moment of $X$ is

$$
m_{r}=\mathbb{E}\left(X^{r}\right)=m_{r, 0}^{\log }=\frac{\alpha}{(r+\alpha)^{2}}[r(1-\lambda)+\alpha] .
$$

Since $\partial m_{r} / \partial \alpha=r[\alpha(\lambda+1)+r(1-\lambda)] /(\alpha+r)^{3}>0, m_{r}$ is increasing with respect to $\alpha$, and since $\partial m_{r} / \partial \lambda=-\alpha \lambda /(\alpha+r)^{2}<0, m_{r}$ is decreasing with respect to $\lambda$.

We can determine the mean and variance of $X$ by

$$
\begin{aligned}
m_{1} & =\frac{\alpha}{(1+\alpha)^{2}}(1-\lambda+\alpha), \quad \sigma^{2}=m_{2}-\left(m_{1}\right)^{2} \\
& =\frac{\alpha}{(2+\alpha)^{2}}[2(1-\lambda)+\alpha]-\frac{\alpha^{2}}{(1+\alpha)^{4}}(1-\lambda+\alpha)^{2} .
\end{aligned}
$$

The coefficient of variation can be calculated as $C V=\sigma / m_{1}$. 
Remark. Based on the alternative formula: $m_{r}=r \int_{0}^{+\infty} x^{r-1}\left(1-F_{\mathrm{LP}}\right.$ $(x ; \alpha, \lambda)) d x$ and the relation in Equation (4), we have

$$
\begin{aligned}
m_{r} & =r \int_{0}^{1} x^{r-1}\left(1-\frac{x}{\alpha} f_{\mathrm{LP}}(x ; \alpha, \lambda)-\lambda x^{\alpha}\right) d x \\
& =r \int_{0}^{1} x^{r-1} d x-\frac{r}{\alpha} \int_{0}^{1} x^{r} f_{\mathrm{LP}}(x ; \alpha, \lambda) d x-\lambda r \int_{0}^{1} x^{\alpha+r-1} d x \\
& =1-\frac{r}{\alpha} m_{r}-\frac{\lambda r}{\alpha+r} .
\end{aligned}
$$

By solving this equation with respect to $m_{r}$, we find the expression of $m_{r}$.

- By the exponential series expansion, the moment generating function of $X$ defined by $M(t)=\mathbb{E}\left(e^{t X}\right)$, can be expanded as

$$
M(t)=\sum_{k=0}^{+\infty} \frac{t^{k}}{k !} m_{k}=\sum_{k=0}^{+\infty} \frac{t^{k}}{k !} \frac{\alpha}{(k+\alpha)^{2}}[k(1-\lambda)+\alpha]
$$

- By the binomial formula, the $r$-th central moment of $X$ follows as

$$
\begin{aligned}
m_{r}^{c} & =\mathbb{E}\left(\left(X-m_{1}\right)^{r}\right)=\left.\sum_{k=0}^{r}\right|_{k} ^{r}(-1)^{k}\left(m_{1}\right)^{k} m_{r-k} \\
& =\sum_{k=0}^{r}{ }_{k}^{r}{ }_{k}(-1)^{k} \frac{\alpha^{k+1}}{(1+\alpha)^{2 k}(r-k+\alpha)^{2}}(1-\lambda+\alpha)^{k}[(r-k)(1-\lambda)+\alpha] .
\end{aligned}
$$

With this quantity, we define the general coefficient of $X$ by $\mathfrak{E}_{r}=m_{r}^{c} / \sigma^{r}$. Using $r=3$ and $r=4$, we describe the moment skewness and kurtosis of $X$, respectively. 
Figure 5 presents some curves of $\mathfrak{E}_{3}$ with respect to $\alpha$ for several values of $\lambda$.

$$
\mathrm{C}_{3}(\lambda=0.2)
$$
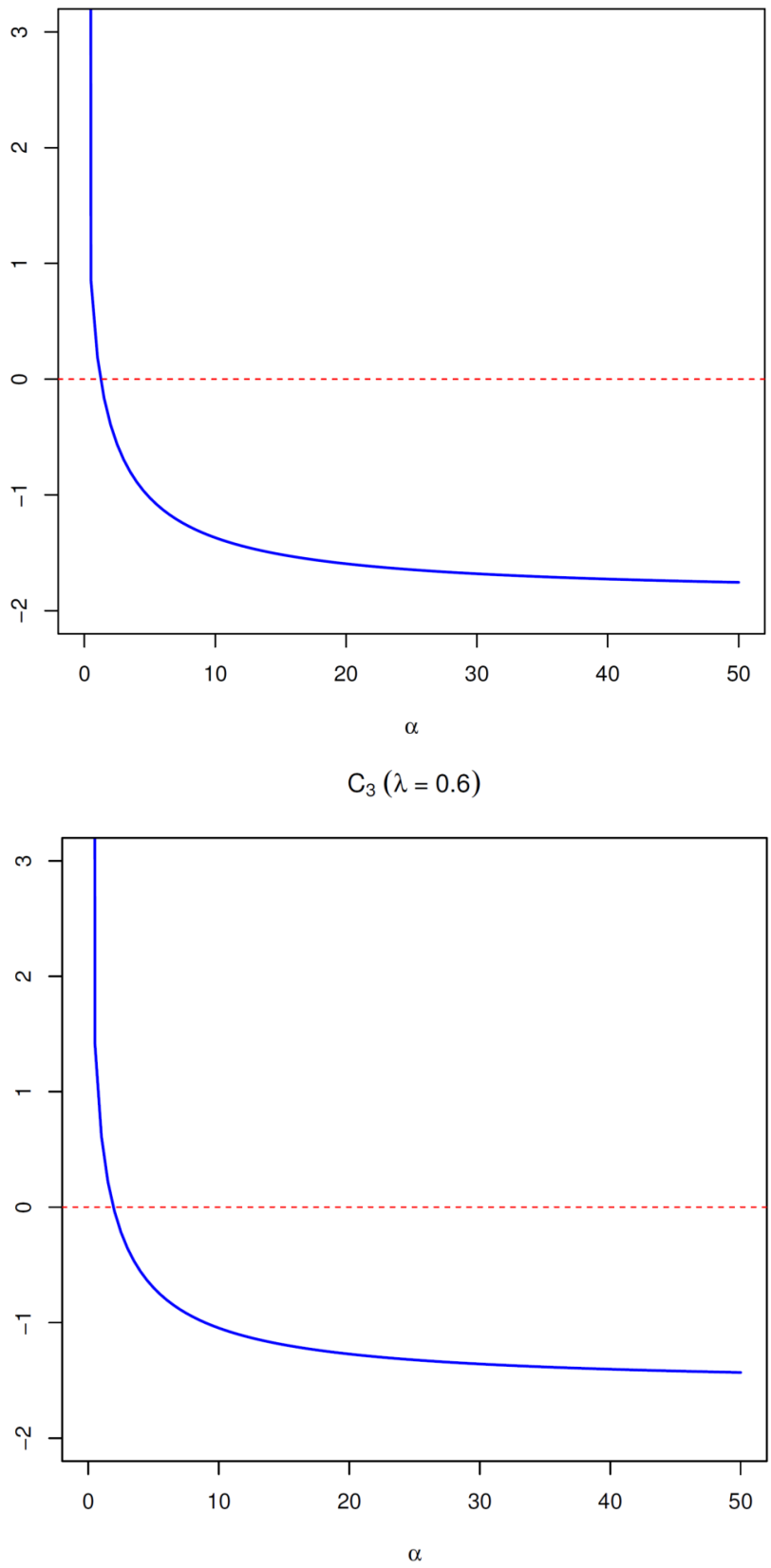


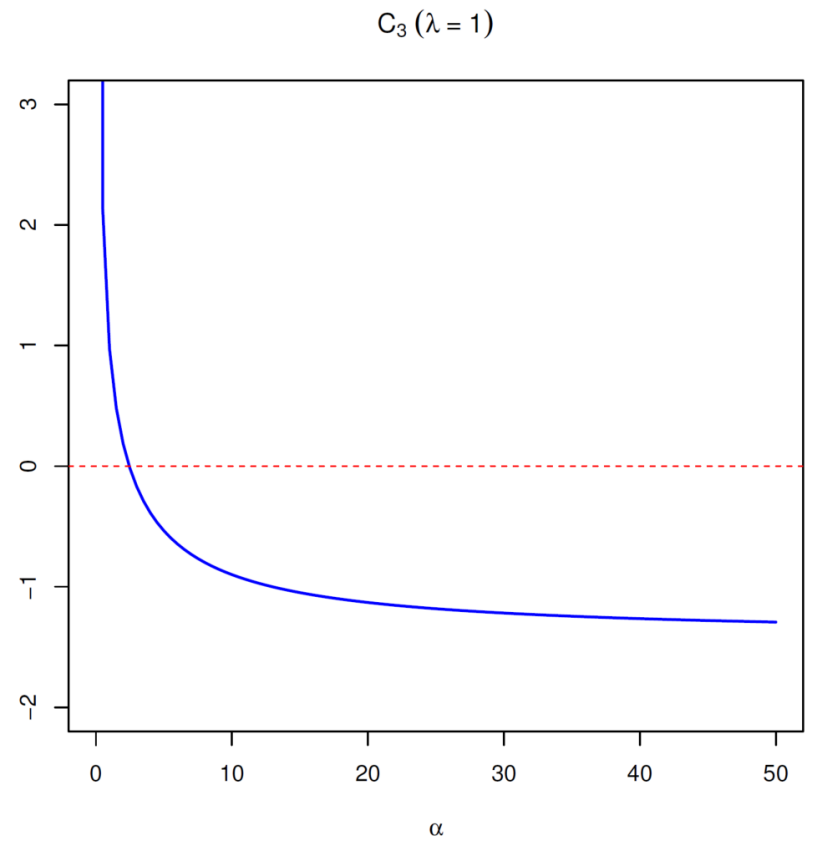

Figure 5. Curves for the moment skewness $\mathfrak{E}_{3}$ with respect to $\alpha$ for several values of $\lambda$.

From Figure 5, we see that $\mathfrak{E}_{3}$ is a purely decreasing function with respect to $\alpha$ and can be either negative or positive, implying that the LP distribution can be left or right skewed. 
Figure 6 shows some curves of $\mathfrak{E}_{4}$ with respect to $\alpha$ for several values of $\lambda$.
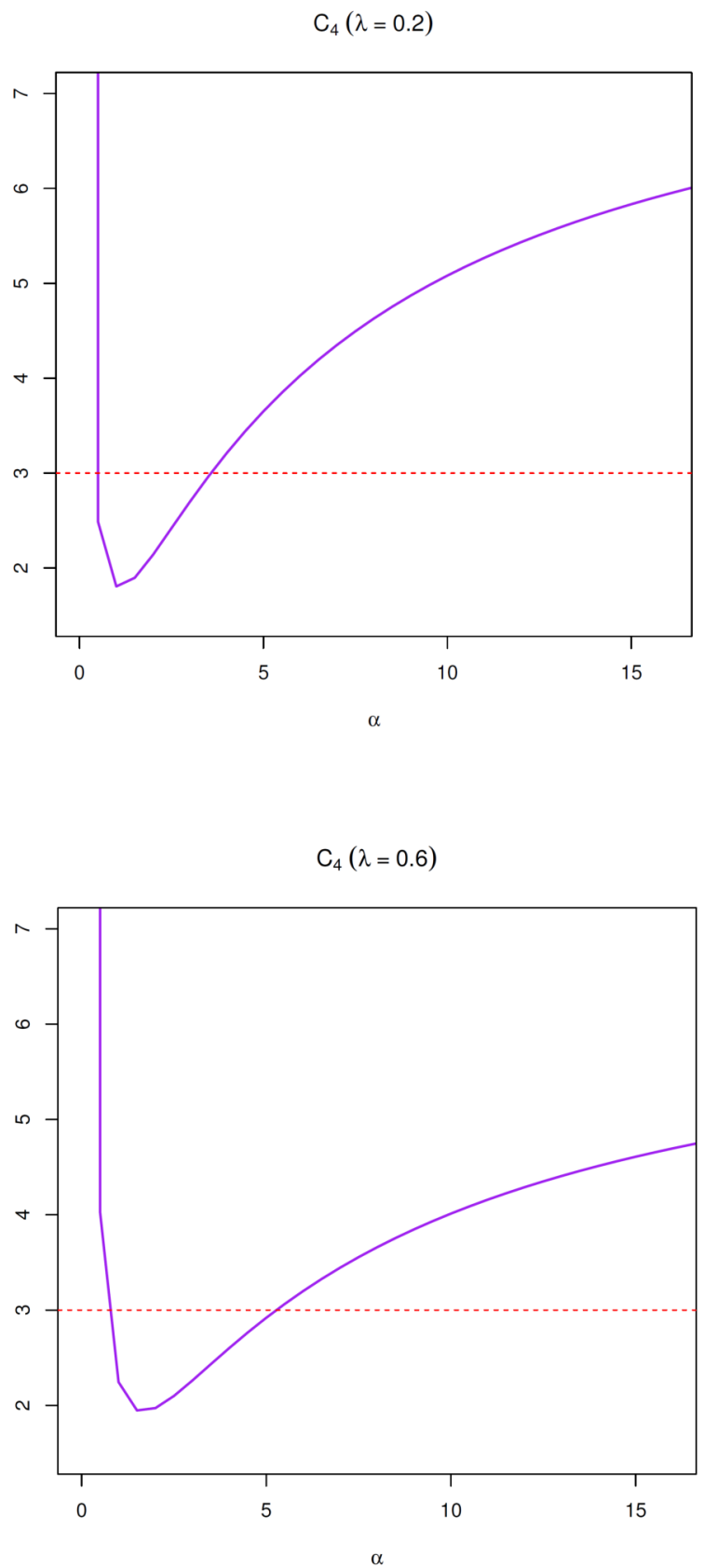
$\mathrm{C}_{4}(\lambda=1)$

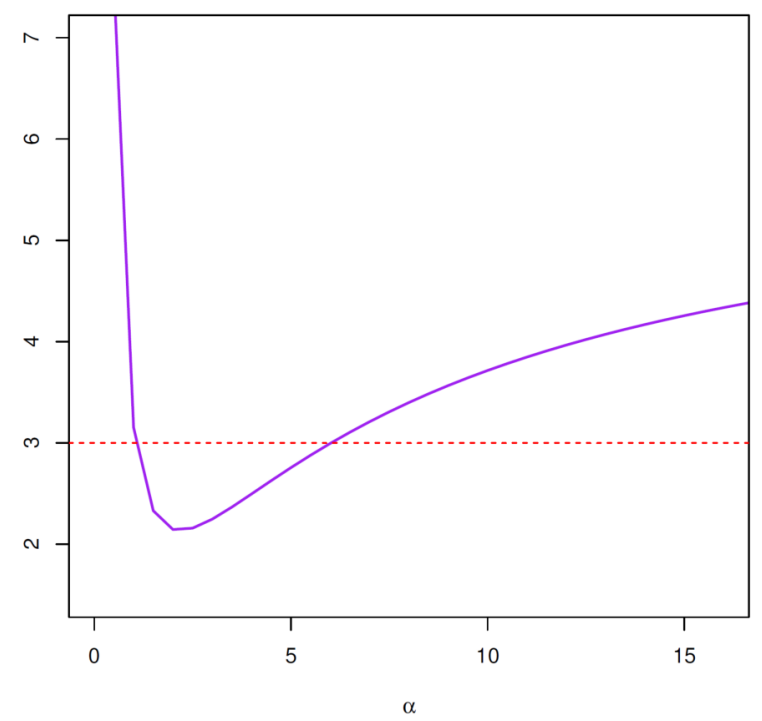

Figure 6. Curves for the moment kurtosis $\mathfrak{E}_{4}$ with respect to $\alpha$ for several values of $\lambda$.

From Figure 6, we note that $\mathfrak{E}_{4}$ is a non-monotonic V-shaped function. Furthermore, it can be less than 3, equal to 3, or greater than 3, indicating that the LP distribution is platykurtic, mesokurtic, or leptokurtic, respectively.

- For any integer $v$ such that $v<\alpha$, the $v$-th negative moment of $X$ is

$$
m_{v}^{-}=\mathbb{E}\left(X^{-v}\right)=m_{-v, 0}^{\log }=\frac{\alpha}{(\alpha-v)^{2}}[\alpha-v(1-\lambda)]
$$

In particular, for $\alpha>1$, the harmonic mean of $X$ can be expressed as

$$
m_{1}^{-}=\mathbb{E}\left(X^{-1}\right)=\frac{\alpha}{(\alpha-1)^{2}}(\alpha-1+\lambda) .
$$

The variance and other moment measures of $Y=X^{-1}$ can be calculated in a similar manner. 
- The $s$-th logarithmic moment of $X$ is

$$
m_{s}^{\log }=\mathbb{E}\left([\log (X)]^{s}\right)=m_{0, s}^{\log }=\frac{(-1)^{s} s !}{\alpha^{s}}(1+\lambda s)
$$

In particular, the geometric mean of $X$ is given by

$$
m_{1}^{\log }=\mathbb{E}(\log (X))=-\frac{1+\lambda}{\alpha} .
$$

In a similar way, the variance and other moment measures of $Y=\log (X)$ can be calculated.

As a result, the most important measures of the LP distribution can be expressed in a straight-forward manner, which will be of interest in future research.

We end this part by investigating the incomplete moments of the LP distribution, which appear in several functions and measures of importance.

Proposition 4.2. Let $r$ be an integer such that $r>-\alpha, s$ be a positive integer, $t \in[0,1]$ and be $X$ be a random variable with the LP distribution. We define the truncated random variable $X_{t}$ by $X_{t}=X$ if the event $\{X \leq t\}$ is realized, and $X_{t}=0$ elsewhere. Then, the $r$-th incomplete moment of $X$ is given as the r-th ordinary moment of $X_{t}$, and we have

$$
m_{r}(t)=\mathbb{E}\left(X_{t}^{r}\right)=\frac{\alpha}{r+\alpha} t^{r+\alpha}\left[\frac{r(1-\lambda)+\alpha}{r+\alpha}-\lambda \alpha \log (t)\right] .
$$

Proof. We have

$$
\begin{aligned}
m_{r}(t) & =\int_{-\infty}^{t} x^{r} f_{\mathrm{LP}}(x ; \alpha, \lambda) d x=\alpha \int_{0}^{t} x^{r+\alpha-1}[1-\lambda-\lambda \alpha \log (x)] d x \\
& =\alpha\left[(1-\lambda) \int_{0}^{t} x^{r+\alpha-1} d x-\lambda \alpha \int_{0}^{t} x^{r+\alpha-1} \log (x) d x\right] .
\end{aligned}
$$


By applying an integration by part, we get

$$
\begin{aligned}
\int_{0}^{t} x^{r+\alpha-1} \log (x) d x= & \left.\frac{x^{r+\alpha}}{r+\alpha} \log (x)\right|_{0} ^{t} \\
& -\int_{0}^{t} \frac{x^{r+\alpha}}{r+\alpha} \frac{1}{x} d x=\frac{t^{r+\alpha}}{r+\alpha}\left(\log (t)-\frac{1}{r+\alpha}\right) .
\end{aligned}
$$

Therefore

$$
\begin{aligned}
m_{r}(t) & =\alpha\left[(1-\lambda) \frac{t^{r+\alpha}}{r+\alpha}-\frac{\lambda \alpha t^{r+\alpha}}{r+\alpha}\left(\log (t)-\frac{1}{r+\alpha}\right)\right] \\
& =\frac{\alpha}{r+\alpha} t^{r+\alpha}\left[\frac{r(1-\lambda)+\alpha}{r+\alpha}-\lambda \alpha \log (t)\right] .
\end{aligned}
$$

The desired result is obtained.

We obviously re-obtain the $r$-th ordinary moments of $X$ by taking $t=1$ in Proposition 4.2. Also, the first incomplete moment of $X$ is given by

$$
m_{1}(t)=\frac{\alpha}{r+\alpha} t^{1+\alpha}\left[1-\frac{\lambda}{1+\alpha}-\lambda \alpha \log (t)\right]
$$

From this, the following moment-type measures can be expressed.

- The mean deviation of $X$ around the mean is given as

$$
\begin{aligned}
& \mathfrak{D}\left(m_{1}\right)=\mathbb{E}\left(\left|X-m_{1}\right|\right)=2 m_{1} F_{\mathrm{LP}}\left(m_{1} ; \alpha, \lambda\right)-2 m_{1}\left(m_{1}\right) \\
& =\frac{2}{1+\alpha}\left(\frac{\alpha}{(1+\alpha)^{2}}(1-\lambda+\alpha)\right)^{\alpha+1}\left[1-\lambda \alpha \log \left(\frac{\alpha}{(1+\alpha)^{2}}(1-\lambda+\alpha)\right)+\frac{\alpha \lambda}{1+\alpha}\right] .
\end{aligned}
$$

- The mean deviation of $X$ around the median $M_{\mathrm{LP}}$ is obtained as

$$
\begin{aligned}
\mathfrak{D}\left(M_{\mathrm{LP}}\right) & =\mathbb{E}\left(\left|X-M_{\mathrm{LP}}\right|\right)=m_{1}-2 m_{1}\left(M_{\mathrm{LP}}\right) \\
& =\frac{\alpha}{(1+\alpha)^{2}}(1-\lambda+\alpha)-2 \frac{\alpha}{1+\alpha} M_{\mathrm{LP}}^{1+\alpha}\left[1-\frac{\lambda}{1+\alpha}-\lambda \alpha \log \left(M_{\mathrm{LP}}\right)\right] .
\end{aligned}
$$


- The mean inactivity time of $X$ is indicated as

$$
\begin{aligned}
\mathfrak{M}(t) & =\mathbb{E}(t-X \mid\{X \leq t\})=t-\frac{1}{F_{\mathrm{LP}}(t ; \alpha, \lambda)} m_{1}(t) \\
& =\frac{t}{1+\alpha}\left\{1+\frac{\alpha \lambda}{(1+\alpha)[1-\lambda \alpha \log (t)]}\right\}, \quad t \in(0,1) .
\end{aligned}
$$

- The Bonferroni curve of the LP distribution is given as

$$
\begin{aligned}
\mathfrak{B}(y) & =\frac{m_{1}\left[Q_{\mathrm{LP}}(y ; \alpha, \lambda)\right]}{y m_{1}} \\
& =\frac{1}{y}\left[Q_{\mathrm{LP}}(y ; \alpha, \lambda)\right]^{1+\alpha}\left[1-\frac{\lambda \alpha(1+\alpha)}{1-\lambda+\alpha} \log \left[Q_{\mathrm{LP}}(y ; \alpha, \lambda)\right], \quad y \in(0,1) .\right.
\end{aligned}
$$

Similarly, other income inequality measures can be expressed, such as the Lorenz or Zenga curves.

Applications of superior order incomplete moments are common in reversed residual lifetime analysis, as well as a variety of related measures involving various conditional moments. In this regard, see Ruiz and Navarro [29] and Cordeiro et al. [10].

\subsection{Approximate approach}

An approximate strategy can be used when the expectation of a transformed random variable is difficult to evaluate and needs to be controlled in an analytical or computational way. The following result looks into such a strategy in the context of the LP distribution.

Proposition 4.3. Let $q(x)$ be a function defined on $(0,1)$, and $X$ be a random variable with the LP distribution. We suppose that $m[q]=\mathbb{E}(q(X))$ exists, and the same for $e_{\ell}[q]=\int_{0}^{1} x^{\ell+\alpha-1} q(x) d x$, for all positive integer $\ell$. Subject to convergence of the involved series, for any large integer $\mathfrak{t}$, the following approximation is acceptable: 


$$
m[q] \approx \sum_{k=0}^{\mathfrak{t}} \sum_{\ell=0}^{k} b_{k, \ell} e_{\ell}[q]
$$

where $b_{0,0}=\alpha(1-\lambda)$ and $b_{k, \ell}=\left(\begin{array}{l}k \\ \ell\end{array}\right)(-1)^{\ell}\left(\alpha^{2} \lambda / k\right)$ for $k \geq 1$ and $\ell=0, \ldots, k$.

Proof. Basically, we have

$$
m[q]=\int_{-\infty}^{+\infty} q(x) f_{\mathrm{LP}}(x ; \alpha, \lambda) d x=\int_{0}^{1} q(x)\left\{\alpha x^{\alpha-1}[1-\lambda-\lambda \alpha \log (x)]\right\} d x .
$$

By virtue of the series expansion of the logarithmic function, and the binomial formula, for $x \in(0,1)$, we get

$$
\begin{aligned}
& \alpha x^{\alpha-1}[1-\lambda-\lambda \alpha \log (x)]=\alpha x^{\alpha-1}\left[1-\lambda+\lambda \alpha \sum_{k=1}^{+\infty} \frac{1}{k}(1-x)^{k}\right] \\
& =\alpha x^{\alpha-1}\left[1-\lambda+\lambda \alpha \sum_{k=1}^{+\infty} \frac{1}{k} \sum_{\ell=0}^{k}(k)(-1)^{\ell} x^{\ell}\right]=\sum_{k=0}^{+\infty} \sum_{\ell=0}^{k} b_{k, \ell} x^{\ell+\alpha-1} .
\end{aligned}
$$

Therefore, provided the mathematical validity of the interchange of the sum and integral signs, which depends on the definition of $q(x)$ mainly, we have

$$
m[q]=\sum_{k=0}^{+\infty} \sum_{\ell=0}^{k} b_{k, \ell} \int_{0}^{1} x^{\ell+\alpha-1} q(x) d x \approx \sum_{k=0}^{\mathfrak{t}} \sum_{\ell=0}^{k} b_{k, \ell} e_{\ell}[q]
$$

The proof of Proposition 4.3 is now complete.

In Proposition 4.3, it is implicitely supposed that the expression of $e_{\ell}[q]$ is relatively simple. Hence, because the sums involved are finite, the evaluation of $m[q]$ is manageable and can be used for a variety of mathematical and practical purposes. 


\section{Entropy Analysis}

An entropy measure of a random variable can be thought of as a measure of uncertainty; the higher the entropy, the more chaos there is in the randomness of the associated distribution. Among the most useful entropy measures is the Rényi entropy proposed by Rényi [28]. The Rényi entropy of a random variable $X$ with the LP distribution may be defined as

$$
\mathfrak{E}(\tau)=\frac{1}{1-\tau} \log \left\{\mathbb{E}\left(\left[f_{\mathrm{LP}}(X ; \alpha, \lambda)\right]^{\tau-1}\right)\right\} .
$$

It is commonly assumed that $\tau>0$ and $\tau \neq 1$. In the next result, we express this entropy measure for special values of $\tau$.

Proposition 5.1. Let $\tau$ be a positive integer such that $\tau \geq 2$ and $X$ be a random variable with the LP distribution. Then the Rényi entropy of $X$ is given by

$$
\mathfrak{E}(\tau)=\frac{1}{1-\tau} \log \left\{\sum_{k=0}^{\tau} \frac{\tau !}{(\tau-k) !} \frac{\lambda^{k} \alpha^{k+\tau}(1-\lambda)^{\tau-k}}{(\tau(\alpha-1)+1)^{k+1}}\right\} .
$$

Proof. It follows from the binomial formula and Equation (6) that

$$
\begin{aligned}
\mathbb{E}\left(\left[f_{\mathrm{LP}}(X ; \alpha, \lambda)\right]^{\tau-1}\right) & =\int_{-\infty}^{+\infty}\left[f_{\mathrm{LP}}(x ; \alpha, \lambda)\right]^{\tau} d x \\
& =\int_{0}^{1} \alpha^{\tau} x^{\tau(\alpha-1)}[1-\lambda-\lambda \alpha \log (x)]^{\tau} d x \\
& =\sum_{k=0}^{\tau}\left(\begin{array}{l}
\tau \\
k
\end{array}\right)(-1)^{k} \lambda^{k} \alpha^{k+\tau}(1-\lambda)^{\tau-k} \int_{0}^{1} x^{\tau(\alpha-1)}[\log (x)]^{k} d x \\
& =\sum_{k=0}^{\tau}\left(\begin{array}{l}
\tau \\
k
\end{array}\right)(-1)^{k} \lambda^{k} \alpha^{k+\tau}(1-\lambda)^{\tau-k}(-1)^{k} \frac{k !}{(\tau(\alpha-1)+1)^{k+1}} \\
& =\sum_{k=0}^{\tau} \frac{\tau !}{(\tau-k) !} \frac{\lambda^{k} \alpha^{k+\tau}(1-\lambda)^{\tau-k}}{(\tau(\alpha-1)+1)^{k+1}} .
\end{aligned}
$$

We end the proof by composition with the appropriate weighted logarithmic function. 
In the precise setting of the proof, the binomial formula can not be extended in the case of any real number $\tau$; expressing the Rényi entropy in this case remains a mathematical challenge. Note that the sum in Proposition 5.1 is finite; the Rényi entropy of the LP distribution may be calculated relatively easily.

\section{Order Statistics}

This section establishes some properties of the order statistics of the LP distribution. The minimum and maximum of random variables are special cases. The general theory can be found in Shahbaz et al. [30].

\subsection{Main functions}

Let $\left(X_{1}, \ldots, X_{n}\right)$ be a $n$-sample from the LP distribution, so the components of this vector are $n$ independent and identically distributed random variables with the LP distribution as common distribution. Now we organize these random variables in increasing order of magnitude to obtain ordered random variables denoted by $X_{1: n}, \ldots, X_{n: n}$. Thus defined, the event $\left\{X_{1: n} \leq X_{2: n} \leq \ldots \leq X_{n: n}\right\}$ is certain. After that, for any $j=1,2, \ldots, n$, the cdf of $X_{j: n}$ can be expressed through a general known formula; it is obtained as

$$
F_{j: n}(x ; \alpha, \lambda)=\sum_{k=j}^{n}\left(\begin{array}{l}
n \\
k
\end{array}\right)\left[F_{\mathrm{LP}}(x ; \alpha, \lambda)\right]^{k}\left[1-F_{\mathrm{LP}}(x ; \alpha, \lambda)\right]^{n-k}, \quad x \in \mathbb{R} .
$$

Moreover, the pdf of $X_{j: n}$ is specified by

$$
f_{j: n}(x ; \alpha, \lambda)=n\left(\begin{array}{c}
n-1 \\
j-1
\end{array}\right) f_{\mathrm{LP}}(x ; \alpha, \lambda)\left[F_{\mathrm{LP}}(x ; \alpha, \lambda)\right]^{j-1}\left[1-F_{\mathrm{LP}}(x ; \alpha, \lambda)\right]^{n-j}, \quad x \in \mathbb{R} .
$$


That is, in an expanded form, we have

$$
F_{j: n}(x ; \alpha, \lambda)=\left\{\begin{array}{cc}
1, & x>1, \\
\sum_{k=j}^{n}\left(\begin{array}{l}
n \\
k
\end{array}\right) x^{\alpha k}[1-\lambda \alpha \log (x)]^{k}\left\{1-x^{\alpha}[1-\lambda \alpha \log (x)]\right\}^{n-k}, & x \in(0,1), \\
0, & x<0,
\end{array}\right.
$$

and

$$
\begin{aligned}
& f_{j: n}(x ; \alpha, \lambda)
\end{aligned}
$$

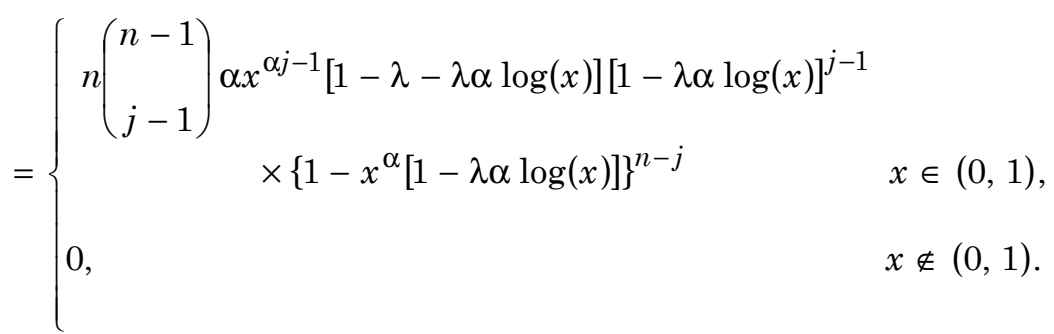

The hrf of $X_{j: n}$ is defined as $h_{j: n}(x ; \alpha, \lambda)=f_{j: n}(x ; \alpha, \lambda) /\left[1-F_{j: n}(x ; \alpha, \lambda)\right]$, $x \in \mathbb{R}$. These functions serve as the foundation for a more in-depth examination of order statistics. To demonstrate this, a moment analysis is shown below.

\subsection{Moment analysis}

The following theorem proposes a sum expression of the $r$-th moment of $X_{j: n}$.

Theorem 6.1. Let $r$ be an integer such that $r>-\alpha, n$ and $j$ be two positive integers such that $j \leq n$, and $X_{j: n}$ be the $j$-th order statistics of an $n$-sample from the LP distribution. Then the r-th ordinary moment of $X_{j: n}$ is given as

$$
m_{j: n, r}^{\text {order }}=\mathbb{E}\left(X_{j: n}^{r}\right)=\sum_{k=0}^{n-j} \sum_{\ell=0}^{k+j-1} \phi_{j: n, k, \ell} \frac{1}{(r+\alpha(k+j))^{\ell+1}}\left[1-\lambda+\frac{\lambda \alpha(\ell+1)}{r+\alpha(k+j)}\right],
$$


where

$$
\phi_{j: n, k, \ell}=n\left(\begin{array}{c}
n-1 \\
j-1
\end{array}\right)\left(\begin{array}{c}
n-j \\
k
\end{array}\right)\left(\begin{array}{c}
k+j-1 \\
\ell
\end{array}\right)(-1)^{k} \lambda^{\ell} \alpha^{\ell+1} \ell !
$$

Proof. First, we have

$$
m_{j: n, r}^{\text {order }}=\int_{-\infty}^{+\infty} x^{r} f_{j: n}(x ; \alpha, \lambda) d x .
$$

Let us now decompose $f_{j: n}(x ; \alpha, \lambda)$ in a suitable way. It follows from the binomial formula that

$$
\begin{aligned}
{[1-\lambda \alpha \log (x)]^{j-1}\left\{1-x^{\alpha}[1-\lambda \alpha \log (x)]\right\}^{n-j} } \\
\quad=\sum_{k=0}^{n-j}\left(\begin{array}{c}
n-j \\
k
\end{array}\right)(-1)^{k} x^{\alpha k}[1-\lambda \alpha \log (x)]^{k+j-1} \\
=\sum_{k=0}^{n-j} \sum_{\ell=0}^{k+j-1}\left(\begin{array}{c}
n-j \\
k
\end{array}\right)\left(\begin{array}{c}
k+j-1 \\
\ell
\end{array}\right)(-1)^{k+\ell} \lambda^{\ell} \alpha^{\ell} x^{\alpha k}[\log (x)]^{\ell} .
\end{aligned}
$$

Therefore

$$
\begin{aligned}
f_{j: n}(x ; \alpha, \lambda)= & n\left(\begin{array}{c}
n-1 \\
j-1
\end{array}\right) \sum_{k=0}^{n-j} \sum_{\ell=0}^{k+j-1}\left(\begin{array}{c}
n-j \\
k
\end{array}\right)\left(\begin{array}{c}
k+j-1 \\
\ell
\end{array}\right)(-1)^{k+\ell} \lambda^{\ell} \alpha^{\ell+1} \\
& \times\left\{(1-\lambda) x^{\alpha(k+j)-1}[\log (x)]^{\ell}-\lambda \alpha x^{\alpha(k+j)-1}[\log (x)]^{\ell+1}\right\} .
\end{aligned}
$$

It follows from this decomposition and Equation (6) that

$$
\begin{aligned}
m_{j: n, r}^{\text {order }}= & n\left(\begin{array}{c}
n-1 \\
j-1
\end{array}\right) \sum_{k=0}^{n-j} \sum_{\ell=0}^{k+j-1}\left(\begin{array}{c}
n-j \\
k
\end{array}\right)\left(\begin{array}{c}
k+j-1 \\
\ell
\end{array}\right)(-1)^{k+\ell} \lambda^{\ell} \alpha^{\ell+1} \\
& \times\left\{(1-\lambda) \int_{0}^{1} x^{r+\alpha(k+j)-1}[\log (x)]^{\ell} d x-\lambda \alpha \int_{0}^{1} x^{r+\alpha(k+j)-1}[\log (x)]^{\ell+1} d x\right\}
\end{aligned}
$$




$$
\begin{aligned}
= & n\left(\begin{array}{c}
n-1 \\
j-1
\end{array}\right) \sum_{k=0}^{n-j} \sum_{\ell=0}^{k+j-1}\left(\begin{array}{c}
n-j \\
k
\end{array}\right)\left(\begin{array}{c}
k+j-1 \\
\ell
\end{array}\right)(-1)^{k+\ell} \lambda^{\ell} \alpha^{\ell+1} \\
& \times\left\{(1-\lambda)(-1)^{\ell} \frac{\ell !}{(r+\alpha(k+j))^{\ell+1}}-\lambda \alpha(-1)^{\ell+1} \frac{(\ell+1) !}{(r+\alpha(k+j))^{\ell+2}}\right\} \\
= & \sum_{k=0}^{n-j} \sum_{\ell=0}^{k+j-1} \phi_{j: n, k, \ell} \frac{1}{(r+\alpha(k+j))^{\ell+1}}\left[1-\lambda+\frac{\lambda \alpha(\ell+1)}{r+\alpha(k+j)}\right] .
\end{aligned}
$$

We get the desired result.

By taking $j=n=1$, we can verify that $m_{j: n, r}^{\text {order }}=m_{r}$. One can remark that the sums involved in Proposition 6.1 are finite; the ordinary moments of order statistics are computable at no cost.

Also, we can calculate the mean and variance of $X_{j: n}$; they can be obtained as

$$
\begin{aligned}
m_{1}^{\text {order }} & =m_{j: n, 1}^{\text {order }} \\
& =\sum_{k=0}^{n-j} \sum_{\ell=0}^{k+j-1} \phi_{j: n, k, \ell} \frac{1}{(1+\alpha(k+j))^{\ell+1}}\left[1-\lambda+\frac{\lambda \alpha(\ell+1)}{1+\alpha(k+j)}\right],
\end{aligned}
$$

and

$$
\begin{aligned}
\left(\sigma^{\text {order }}\right)^{2}= & m_{j: n, 2}^{\text {order }}-\left(m_{j: n, 1}^{\text {order }}\right)^{2} \\
= & \sum_{k=0}^{n-j} \sum_{\ell=0}^{k+j-1} \phi_{j: n, k, \ell} \frac{1}{(2+\alpha(k+j))^{\ell+1}}\left[1-\lambda+\frac{\lambda \alpha(\ell+1)}{2+\alpha(k+j)}\right] \\
& -\left\{\sum_{k=0}^{n-j} \sum_{\ell=0}^{k+j-1} \phi_{j: n, k, \ell} \frac{1}{(1+\alpha(k+j))^{\ell+1}}\left[1-\lambda+\frac{\lambda \alpha(\ell+1)}{1+\alpha(k+j)}\right]\right\}^{2} .
\end{aligned}
$$

The result below proposes a recurrence relation between the ordinary moments of order statistics. 
Theorem 6.2. Let $r$ be an integer such that $r>-\alpha, n$ and $j$ be two positive integers such that $3 \leq j \leq n$, and $X_{j: n}$ be the $j$-th order statistics of an $n$-sample from the LP distribution. Then the following recurrence relation holds:

$$
\begin{aligned}
m_{j: n, r}^{\text {order }}= & {\left[1+\frac{r}{\alpha(j-1)}\right] m_{j-1: n, r}^{\text {order }} } \\
& +\frac{\lambda n r}{(j-1)(\alpha+r)}\left(m_{j-1: n-1, \alpha+r}^{\text {order }}-m_{j-2: n-1, \alpha+r}^{\text {order }}\right)
\end{aligned}
$$

Note that $\alpha+r$ is not necessary an integer, but the formal expectation definitions of $m_{j-1: n-1, \alpha+r}^{\text {order }}-m_{j-2: n-1, \alpha+r}^{\text {order }}$ are still valid in the mathematical sense.

Proof. Thanks to (Shahbaz et al. [30], Theorem 2.10, Equation (2.57)) applied to the context of the LP distribution, we have

$$
\begin{aligned}
m_{j: n, r}^{\text {order }}-m_{j-1: n, r}^{\text {order }}= & n\left(\begin{array}{l}
n-1 \\
j-1
\end{array}\right) \frac{r}{n-j+1} \int_{-\infty}^{+\infty} x^{r-1}\left[F_{\mathrm{LP}}(x ; \alpha, \lambda)\right]^{j-1} \\
& \times\left[1-F_{\mathrm{LP}}(x ; \alpha, \lambda)\right]^{n-j+1} d x .
\end{aligned}
$$

By using the relation in Equation (4), it comes

$$
\begin{aligned}
m_{j: n, r}^{\text {order }}-m_{j-1: n, r}^{\text {order }}= & n\left(\begin{array}{c}
n-1 \\
j-1
\end{array}\right) \frac{r}{n-j+1} \int_{-\infty}^{+\infty} x^{r-1}\left[F_{\mathrm{LP}}(x ; \alpha, \lambda)\right]^{j-2} \\
& \times\left[1-F_{\mathrm{LP}}(x ; \alpha, \lambda)\right]^{n-j+1} F_{\mathrm{LP}}(x ; \alpha, \lambda) d x \\
= & \frac{n}{\alpha}\left(\begin{array}{c}
n-1 \\
j-1
\end{array}\right) \frac{r}{n-j+1} \int_{-\infty}^{+\infty} x^{r}\left[F_{\mathrm{LP}}(x ; \alpha, \lambda)\right]^{j-2} \\
& \times\left[1-F_{\mathrm{LP}}(x ; \alpha, \lambda)\right]^{n-j+1} f_{\mathrm{LP}}(x ; \alpha, \lambda) d x
\end{aligned}
$$




$$
\begin{aligned}
& +\lambda n\left(\begin{array}{l}
n-1 \\
j-1
\end{array}\right) \frac{r}{n-j+1} \int_{-\infty}^{+\infty} x^{\alpha+r-1}\left[F_{\mathrm{LP}}(x ; \alpha, \lambda)\right]^{j-2} \\
& \times\left[1-F_{\mathrm{LP}}(x ; \alpha, \lambda)\right]^{n-j+1} d x .
\end{aligned}
$$

Now, from Equation (7), we have

$$
\begin{aligned}
\int_{-\infty}^{+\infty} x^{r}\left[F_{\mathrm{LP}}(x ; \alpha, \lambda)\right]^{j-2}\left[1-F_{\mathrm{LP}}(x ; \alpha, \lambda)\right]^{n-j+1} f_{\mathrm{LP}}(x ; \alpha, \lambda) d x & \\
= & \frac{1}{n\left(\begin{array}{c}
n-1 \\
j-2
\end{array}\right)} m_{j-1: n, r}^{\text {order }} .
\end{aligned}
$$

Moreover, by adapting Equation (9), we get

$$
\begin{aligned}
\int_{-\infty}^{+\infty} & x^{\alpha+r-1}\left[F_{\mathrm{LP}}(x ; \alpha, \lambda)\right]^{j-2}\left[1-F_{\mathrm{LP}}(x ; \alpha, \lambda)\right]^{n-j+1} d x \\
& =\frac{1}{(n-1)\left(\begin{array}{l}
n-2 \\
j-2
\end{array}\right)(\alpha+r) /(n-j+1)}\left(m_{j-1: n-1, \alpha+r}^{\text {order }}-m_{j-2: n-1, \alpha+r}^{\text {order }}\right) .
\end{aligned}
$$

By putting the above equalities together, after simplifications of the constant terms, we obtain

$$
\begin{aligned}
m_{j: n, r}^{\text {order }}-m_{j-1: n, r}^{\text {order }}= & \frac{r}{\alpha(j-1)} m_{j-1: n, r}^{\text {order }} \\
& +\frac{\lambda n r}{(j-1)(\alpha+r)}\left(m_{j-1: n-1, \alpha+r}^{\text {order }}-m_{j-2: n-1, \alpha+r}^{\text {order }}\right)
\end{aligned}
$$

The stated result is established.

The L-moments of a random variable are defined by a linear combination of ordinary moments of the associated order statistics. In our setting, for any integer $r>-\alpha$ and a random variable $X$ with the LP distribution, the $r$-th L-moment of $X$ is specified by

$$
\mathfrak{U}_{r}=\frac{1}{r} \sum_{k=0}^{r-1}\left(\begin{array}{c}
r-1 \\
k
\end{array}\right)(-1)^{k} m_{r-k: r, 1}^{\text {order }} .
$$


The L-moments can be used to summarize the shape of a distribution in a similar way to conventional moments. Based on the L-moments, we can define various L-moment measures, such as the L-coefficient of variation, L-skewness and L-kurtosis. For more information on these measures, we can refer to Hosking [18].

\section{Measure of Reliability}

Many physics and engineering applications, such as strength loss and system breakdown, need a valuable measure of reliability. In particular, when a component of a system is exposed to random stress and is subjected to random strength, the probability that the stress exceeds the strength is of importance. In general, stress is modelled by a random variable $X_{2}$, strength is modelled by a random variable $X_{1}$, and the considered probability measure can be formulated as $\mathfrak{R}=\mathbb{P}\left(X_{2} \leq X_{1}\right)$. It is a key ingredient of the stress-strength model. We may refer to Kotz et al. [22] for all the possible uses of this measure. In the following result, we determine its expression in the context of the LP distribution.

Theorem 7.1. Let $X_{1}$ and $X_{2}$ be two independent random variables with the $\operatorname{LP}\left(\alpha_{1}, \lambda_{1}\right)$ and $L P\left(\alpha_{2}, \lambda_{2}\right)$ distributions, respectively. Then, we have

$$
\begin{aligned}
& \alpha_{1} \alpha_{2} \lambda_{2}\left[\alpha_{1} \lambda_{1}+\alpha_{1}+\alpha_{2}\left(1-\lambda_{1}\right)\right] \\
& \mathfrak{R}=\mathbb{P}\left(X_{2} \leq X_{1}\right)=\frac{+\alpha_{1}\left(\alpha_{1}+\alpha_{2}\right)\left[\alpha_{1}+\alpha_{2}\left(1-\lambda_{1}\right)\right]}{\left(\alpha_{1}+\alpha_{2}\right)^{3}} .
\end{aligned}
$$


Proof. The proof is mainly based on calculation. By using the independence and distributions of $X_{1}$ and $X_{2}$, we obtain

$$
\begin{aligned}
\Re=\mathbb{P}\left(X_{2} \leq X_{1}\right)= & \int_{-\infty}^{+\infty} F_{\mathrm{LP}}\left(x ; \alpha_{2}, \lambda_{2}\right) f_{\mathrm{LP}}\left(x ; \alpha_{1}, \lambda_{1}\right) d x \\
= & \alpha_{1} \int_{0}^{1} x^{\alpha_{1}+\alpha_{2}-1}\left[1-\lambda_{2} \alpha_{2} \log (x)\right]\left[1-\lambda_{1}-\lambda_{1} \alpha_{1} \log (x)\right] d x \\
= & \alpha_{1} \int_{0}^{1} x^{\alpha_{1}+\alpha_{2}-1}\left\{\left(1-\lambda_{1}\right)-\left[\left(1-\lambda_{1}\right) \lambda_{2} \alpha_{2}+\lambda_{1} \alpha_{1}\right] \log (x)\right. \\
& \left.+\lambda_{1} \lambda_{2} \alpha_{1} \alpha_{2}[\log (x)]^{2}\right\} d x \\
= & \alpha_{1}\left(1-\lambda_{1}\right) \int_{0}^{1} x^{\alpha_{1}+\alpha_{2}-1} d x-\alpha_{1}\left[\left(1-\lambda_{1}\right) \lambda_{2} \alpha_{2}+\lambda_{1} \alpha_{1}\right] \\
& \times \int_{0}^{t} x^{\alpha_{1}+\alpha_{2}-1} \log (x) d x \\
& +\lambda_{1} \lambda_{2} \alpha_{1}^{2} \alpha_{2} \int_{0}^{1} x^{\alpha_{1}+\alpha_{2}-1}[\log (x)]^{2} d x
\end{aligned}
$$

By applying Equation (6), we get

$$
\begin{aligned}
\Re=\alpha_{1}\left(1-\lambda_{1}\right) \frac{1}{\alpha_{1}+\alpha_{2}} & +\alpha_{1}\left[\left(1-\lambda_{1}\right) \lambda_{2} \alpha_{2}+\lambda_{1} \alpha_{1}\right] \frac{1}{\left(\alpha_{1}+\alpha_{2}\right)^{2}} \\
& +\lambda_{1} \lambda_{2} \alpha_{1}^{2} \alpha_{2} \frac{2}{\left(\alpha_{1}+\alpha_{2}\right)^{3}} \\
= & \frac{\alpha_{1} \alpha_{2} \lambda_{2}\left[\alpha_{1} \lambda_{1}+\alpha_{1}+\alpha_{2}\left(1-\lambda_{1}\right)\right]}{+\alpha_{1}\left(\alpha_{1}+\alpha_{2}\right)\left[\alpha_{1}+\alpha_{2}\left(1-\lambda_{1}\right)\right]}
\end{aligned}
$$

The intended result is achieved. 
Based on Theorem 7.1, the analysis of stress-strength LP model is conceivable. In particular, the estimation of $\alpha_{1}, \lambda_{1}, \alpha_{2}$ and $\lambda_{2}$ yields the estimation of $\mathfrak{R}$ by the substitution approach. Without surprise, when $X_{1}$ and $X_{2}$ are identically distributed, we get $\mathfrak{R}=1 / 2$.

\section{Some Distributional Results}

The LP distribution can be modified to create new distributions with a diverse number of parameters and support. This section presents some of them.

Let us consider a random variable $X$ with the $\operatorname{LP}(\alpha, \lambda)$ distribution.

- Basically, the random variable $Y=X^{\alpha}$ follows the $L P(1, \lambda)$ distribution.

- Let $Y=\beta X$ with $\beta>0$. Then the cdf of $Y$ is determined by

$$
\dot{F}(x ; \alpha, \beta, \lambda)=\left\{\begin{array}{lc}
1, & x \geq \beta, \\
\left(\frac{x}{\beta}\right)^{\alpha}[1+\lambda \alpha \log (\beta)-\lambda \alpha \log (x)], & x \in(0, \beta), \\
0, & x \leq 0 .
\end{array}\right.
$$

It is a three-parameter tuned upper bound variant of the LP distribution. We can use it for the analysis of bounded data beyond the unit interval.

- Let $Y=\beta / X$ with $\beta>0$. Then $Y$ has the following cdf:

$$
\ddot{F}(x ; \alpha, \beta, \lambda)= \begin{cases}1-\left(\frac{\beta}{x}\right)^{\alpha}[1+\lambda \alpha \log (x)-\lambda \alpha \log (\beta)], & x>\beta, \\ 0, & x \leq \beta .\end{cases}
$$

It corresponds to a tuning lower bound on a special variant of the LP distribution. 
- Let $Y=1-X$. Then the cdf of $Y$ is given as

$$
\widetilde{F}(x ; \alpha, \lambda)=\left\{\begin{array}{lc}
1, & x \geq 1, \\
1-(1-x)^{\alpha}[1-\lambda \alpha \log (1-x)], & x \in(0,1), \\
0, & x \leq 0 .
\end{array}\right.
$$

The related distribution, which proposes a new alternative unit distribution, is called the LP distribution of the second kind.

- Let $Y=-\log (X)$. Then $Y$ has the following cdf:

$$
F^{*}(x ; \alpha, \lambda)= \begin{cases}1-(1+\lambda \alpha x) e^{-\alpha x}, & x>0, \\ 0, & x \leq 0 .\end{cases}
$$

If we take $\lambda=0$, we obtain the cdf of the exponential (E) distribution and, if we take $\lambda=1$, it corresponds to the cdf of the length-biased exponential (LBE) distribution introduced by Dara and Ahmad [11]. In this sense, the associated distribution can be seen as a modern tradeoff between the $\mathrm{E}$ and LBE distributions.

- Let $Y=-\log \left(1-X^{\alpha}\right) / \beta$ with $\beta>0$. Then the cdf of $Y$ is specified by

$$
F^{\ominus}(x ; \beta, \lambda)= \begin{cases}\left(1-e^{-\beta x}\right)\left[1-\lambda \log \left(1-e^{-\beta x}\right)\right], & x>0, \\ 0, & x \leq 0 .\end{cases}
$$

It represents a two-parameter lifetime distribution that has never been described before in the literature. It is like a modern-day variant of the exponential distribution. 
- Let $Y=(1 / \beta)(1 / X-1)$ with $\beta>0$. Then the cdf of $Y$ is given as

$$
\check{F}(x ; \alpha, \beta, \lambda)= \begin{cases}1-(1+\beta x)^{-\alpha}[1+\lambda \log (1+\beta x)], & x>0, \\ 0, & x \leq 0 .\end{cases}
$$

If we take $\lambda=0$, it corresponds to the cdf of the Lomax distribution introduced by Lomax [24]. The associated distribution thus constitutes a new three-parameter generalization.

- Let $Y=\log (X /(1-X))$ corresponding to the logistic transformation of $X$. Then the cdf of $Y$ is given as

$$
\breve{F}(x ; \alpha, \lambda)=\left(1+e^{-x}\right)^{-\alpha}\left[1+\lambda \alpha \log \left(1+e^{-x}\right)\right], \quad x \in \mathbb{R} .
$$

The cdf of the skew-logistic distribution corresponds to $\lambda=0$. As a result, the proposed cdf defines a generalized skew-logistic distribution that can be used to model characteristics with values spanning the entire real line. To improve its modelling capability, we can add position and scale parameters, and consider the cdf: $\check{F}(x ; \alpha, \lambda, \mu, \sigma)=\check{F}((x-\mu) / \sigma ; \alpha, \lambda)$, with $\mu \in \mathbb{R}$ and $\sigma>0$.

- Let $Y=\sup \left(X_{1}, \ldots, X_{n}\right)$, where $\left(X_{1}, \ldots, X_{n}\right)$ is a $n$-sample from the LP distribution. In terms of order statistics, we have $Y=X_{n: n}$. Then, as a special case of Equation (8), $Y$ has the following cdf:

$$
F^{\sup }(x ; \alpha, n, \lambda)=\left\{\begin{array}{lc}
1, & x \geq 1, \\
x^{\alpha n}[1-\lambda \alpha \log (x)]^{n}, & x \in(0,1), \\
0, & x \leq 0 .
\end{array}\right.
$$

The associated distribution is useful in the setting of the order statistics theory. The cdf remains correct from a mathematical standpoint for every real number $n>0$. 
- Let $Y=\inf \left(X_{1}, \ldots, X_{n}\right)$, where $\left(X_{1}, \ldots, X_{n}\right)$ is an $n$-sample from the LP distribution. In terms of order statistics, we have $Y=X_{1: n}$. Then, as a special case of Equation (8), $Y$ has the following cdf:

$$
F^{\inf }(x ; \alpha, n, \lambda)= \begin{cases}1, & x \geq 1, \\ 1-\left\{1-x^{\alpha}[1-\lambda \alpha \log (x)]\right\}^{n}, & x \in(0,1), \\ 0, & x \leq 0 .\end{cases}
$$

This cdf holds true for every real number $n>0$.

- With an analytical approach, original three-parameter extensions of the LP distribution are possible. As an example, an interesting perspective is offered by the following function:

$$
F^{\nabla}(x ; \alpha, \beta, \lambda)= \begin{cases}1, & x \geq 1, \\ \frac{1}{1+\lambda \log (\beta+1)} x^{\alpha}\left[1+\lambda \log \left(\beta+x^{-\alpha}\right)\right], & x \in(0,1), \\ 0, & x \leq 0,\end{cases}
$$

with $\alpha>0, \beta \geq 0$ and $\lambda \in[0,1]$. Then, one can prove that it is a valid cdf. The LP distribution is obtained by taking $\beta=0$. We call this new unit distribution the three-parameter LP distribution.

- The LP distribution can be used to extend any continuous distribution to reach new modelling perspectives. A simple strategy is described below. Let $G(x ; \zeta)$ be a cdf of a continuous distribution with $\zeta$ as parameter(s), and $Y=G^{-1}(X ; \zeta)$. Then $Y$ has the following cdf:

$$
F_{G}(x ; \alpha, \lambda, \zeta)=G(x ; \zeta)^{\alpha}[1-\lambda \alpha \log (G(x ; \zeta))], \quad x \in \mathbb{R} .
$$

This cdf defines a new family of distributions extending the baseline distribution characterized by $G(x ; \zeta)$. Such a family can be called the LP generated (LP-G) family. Further information about such general families can be found in Cordeiro et al. [10]. 
- On the other hand, the LP distribution can be extended by using existing general families of distributions, such as the exponentiated, Marshall-Olkin, half-logistic, Kumaraswamy, beta, Topp-Leone, Weibull, Burr-type and sine generated families. The details and references are available in Brito et al. [5].

The majority of the above-mentioned distributions may be the subject of independent investigation, with an emphasis on particular implementations. They can also be extended in a variety of ways using the power scheme.

\section{Estimation and Data Applications}

In this section, we focus on the LP model by assuming that the parameters $\alpha$ and $\lambda$ are unknown.

\subsection{Estimation}

We propose to estimate unknown parameters by the maximum likelihood procedure described as follows. Let $x_{1}, \ldots, x_{n}$ be observations selected from the LP distribution. Then, the likelihood function of the LP model for $\alpha$ and $\lambda$ can be written as

$$
\mathfrak{L}(\alpha, \lambda)=\prod_{i=1}^{n} f_{\mathrm{LP}}\left(x_{i} ; \alpha, \lambda\right)=\alpha^{n}\left[\prod_{i=1}^{n} x_{i}\right]^{\alpha-1} \prod_{i=1}^{n}\left[1-\lambda-\lambda \alpha \log \left(x_{i}\right)\right] .
$$

Based on this function, the log-likelihood function for $\alpha$ and $\lambda$ is

$$
\begin{aligned}
\ell(\alpha, \lambda)=\log [\mathfrak{L}(\alpha, \lambda)]=n \log (\alpha) & +(\alpha-1) \sum_{i=1}^{n} \log \left(x_{i}\right) \\
& +\sum_{i=1}^{n} \log \left[1-\lambda-\lambda \alpha \log \left(x_{i}\right)\right] .
\end{aligned}
$$


Then the maximum likelihood estimates (MLEs) of $\alpha$ and $\lambda$, say $\hat{\alpha}$ and $\hat{\lambda}$, are defined by $(\hat{\alpha}, \hat{\lambda})=\arg \max _{(\alpha, \lambda) \in(0,+\infty) \times[0,1]} \ell(\alpha, \lambda)$. We can describe them via an analytical approach based on nonlinear equations, as described as follows. When the log likelihood function is differentiated with respect to $\alpha$ and $\lambda$, the result is

$$
\frac{\partial}{\partial \alpha} \ell(\alpha, \lambda)=\frac{n}{\alpha}+\sum_{i=1}^{n} \log \left(x_{i}\right)-\lambda \sum_{i=1}^{n} \frac{\log \left(x_{i}\right)}{1-\lambda-\lambda \alpha \log \left(x_{i}\right)}
$$

and

$$
\frac{\partial}{\partial \lambda} \ell(\alpha, \lambda)=-\sum_{i=1}^{n} \frac{1+\alpha \log \left(x_{i}\right)}{1-\lambda-\lambda \alpha \log \left(x_{i}\right)}
$$

Equating these partial derivatives to zero yields $\hat{\alpha}$ and $\hat{\lambda}$. These equations, however, can not be solved analytically and must be solved numerically using statistical software. The estimates can be obtained using iterative techniques such as the Newton-Raphson algorithm. In this work, such estimates are obtained by the use of the function nlminb of the $R$ software (see R Core Team [27]).

Then, an estimation of the unknown pdf $f_{\mathrm{LP}}(x ; \alpha, \lambda)$ is given by $\hat{f}_{\mathrm{LP}}(x)=f_{\mathrm{LP}}(x ; \hat{\alpha}, \hat{\lambda})$. We can proceed similarly to estimate other parametric functions related to the LP distribution.

In addition, we can derive the following well-established criteria from $\ell(\hat{\alpha}, \hat{\lambda})$ : The Akaike information criterion (AIC) and Bayesian information criterion (BIC) are defined as

$$
\mathrm{AIC}=-2 \ell(\hat{\alpha}, \hat{\lambda})+2 k, \quad \mathrm{BIC}=-2 \ell(\hat{\alpha}, \hat{\lambda})+k \log (n),
$$

respectively, where $k$ denotes the number of parameters. The AIC and BIC of several models can be calculated; the best model being the one with the smallest AIC and BIC. We may refer to Casella and Berger [6] for the details about the maximum likelihood procedure. 


\subsection{Data applications}

One artificial data set and two real-life data sets are now being examined. We want to show how useful and adaptable the LP model is when it comes to data fitting. We compare the LP and TP models in this regard. We recall that the pdf of the LP distribution is given by Equation (3), and the pdf of the TP distribution is derived from Equation (1) as

$$
f_{\mathrm{TP}}(x ; \alpha, \lambda)= \begin{cases}\alpha x^{\alpha-1}\left(1+\lambda-2 \lambda x^{\alpha}\right), & x \in(0,1), \\ 0, & x \notin(0,1) .\end{cases}
$$

Our applications are presented below.

- The first data set, called Data set 1 , is artificial; it contains $n=100$ values generated from the LP distribution with parameters $\alpha=50$ and $\lambda=1$. The data set is $\{0.9407981,0.9447613,0.9882597,0.9570619$, $0.9289420,0.9862649,0.9377094,0.9785259,0.9689801,0.9701998$, $0.9885426, \quad 0.9630237, \quad 0.9663357, \quad 0.9648558, \quad 0.9980493, \quad 0.9343859$, $0.9586432,0.9960804,0.9718961,0.9491994,0.9388389,0.9439703$, $0.9853174,0.9746523,0.9149421,0.9758234,0.9432352,0.9693834$, $0.9491918, \quad 0.9851612, \quad 0.9656517, \quad 0.9267656, \quad 0.9680509, \quad 0.9723581$, $0.9069665,0.9133886,0.9533500,0.9342400,0.9798710,0.9492754$, $0.9716584, \quad 0.8734544,0.9831862,0.9643272,0.9918818,0.9334234$, $0.9881646, \quad 0.9949953, \quad 0.9214476, \quad 0.9689857, \quad 0.9516179, \quad 0.9333489$, $0.9730641,0.9400146,0.9668169,0.9654974,0.9428985,0.9812006$, $0.9523705,0.9837782,0.9729954,0.9902377,0.9814711,0.9662710$, $0.8789145, \quad 0.9562315, \quad 0.9647483,0.9767500,0.9124355, \quad 0.9561419$, $0.9813736,0.9502237,0.9877244,0.9746159,0.9678454,0.9388364$, $0.9794370, \quad 0.9186319, \quad 0.9919013, \quad 0.9622962,0.9589765,0.8991588$, $0.9511993, \quad 0.9129763,0.9724723,0.9736149, \quad 0.9677164,0.9432212$, $0.9534555,0.9289118,0.9427475,0.9737435,0.9754736,0.9983466$, $0.9634858,0.9646391,0.9869528,0.9941471, \quad 0.9265261, \quad 0.9872700\}$. 
Table 1 provides the mean, median, standard deviation (St. deviation), variance, skewness, kurtosis, minimum and maximum of this data set.

Table 1. Statistical description of Data set 1

\begin{tabular}{|l|l|}
\hline Mean & 0.959092 \\
Median & 0.9651766 \\
St. deviation & 0.02600243 \\
Variance & 0.0006761264 \\
Skewness & -0.9004576 \\
Kurtosis & 3.692149 \\
Minimum & 0.8734544 \\
Maximum & 0.9983466 \\
\hline
\end{tabular}

We remark that the data are left skewed, mesokurtic, and with a very small variance. The mean and median are almost the same.

For these data, we determine the MLEs $\hat{\alpha}$ and $\hat{\lambda}$ of $\alpha$ and $\lambda$, respectively, and standard information criteria. The results are given in Table 2.

Table 2. Values of the MLEs, AIC and BIC for Data set 1

\begin{tabular}{|ccccc|}
\hline Distribution & MLE $\hat{\alpha}$ & MLE $\hat{\lambda}$ & AIC & BIC \\
\hline LP & 46.98128 & 0.9797222 & -462.8919 & -457.6816 \\
TP & 11.86562 & -1 & -437.7862 & -432.5759 \\
\hline
\end{tabular}

As expected, the estimates $\hat{\alpha}$ and $\hat{\lambda}$ are close to the considered parameter values of the LP distribution used to generate the data, which are 50 and 1 , respectively. 
In terms of AIC and BIC, the LP distribution is clearly the best. So a best fit is expected for the related estimated functions in comparison to the estimated functions of the TP distribution. This is visually confirmed in Figure 7 which considers the estimated pdfs: $\hat{f}_{\mathrm{LP}}(x)=f_{\mathrm{LP}}(x ; \hat{\alpha}, \hat{\lambda})$ and $\hat{f}_{\mathrm{TP}}(x)=f_{\mathrm{TP}}(x ; \hat{\alpha}, \hat{\lambda})$.

\section{Fits of Data set 1}

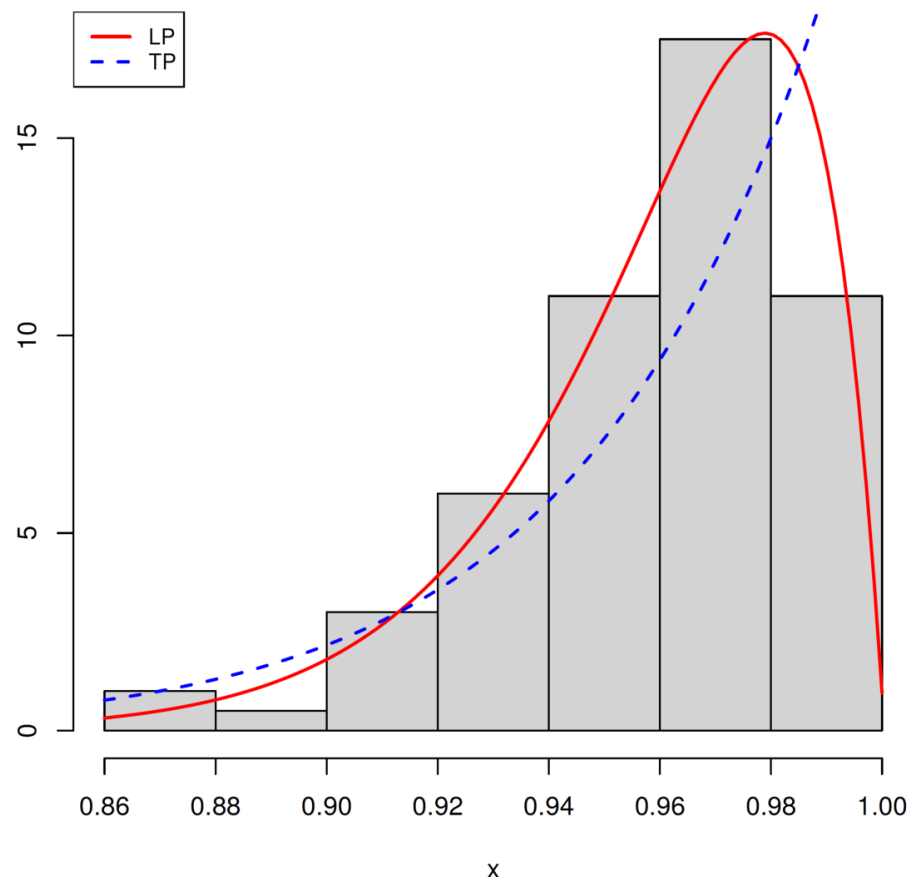

Figure 7. Curves of the estimated pdfs of the LP and TP distributions for Data set 1 .

In Figure 7, we see that the LP distribution has well captured the top of the histogram, contrary to the TP distribution. However, since the data have been generated from the LP distribution, such a result was expected. 
- The second data set, called Data set 2, is extracted from Klein and Moeschberger [21]. It specifies the time it takes for kidney dialysis patients to become infected in months. The data set is: $\{2.5,2.5,3.5,3.5$, $3.5,4.5,5.5,6.5,6.5,7.5,7.5,7.5,7.5,8.5,9.5,10.5,11.5,12.5,12.5,13.5$, $14.5,14.5,21.5,21.5,22.5,22.5,25.5,27.5\}$. We perform a "unit range" operation on these data by dividing them by 28 .

As a basic analysis, Table 3 provides a statistical summary of the unit data set.

Table 3. Statistical description of Data set 2

\begin{tabular}{|l|l|}
\hline Mean & 0.4043367 \\
Median & 0.3214286 \\
St. deviation & 0.2647846 \\
Variance & 0.07011088 \\
Skewness & 0.7650467 \\
Kurtosis & 2.421883 \\
Minimum & 0.08928571 \\
Maximum & 0.9821429 \\
\hline
\end{tabular}

We remark that the data are right skewed, platykurtic and with a wide range of values between $(0,1)$.

For these data, we determine the MLEs $\hat{\alpha}$ and $\hat{\lambda}$ of $\alpha$ and $\lambda$, respectively, and standard information criteria. The results are given in Table 4.

Table 4. Values of the MLEs, AIC and BIC for Data set 2

\begin{tabular}{|ccccc|}
\hline Distribution & MLE $\hat{\alpha}$ & MLE $\hat{\lambda}$ & AIC & BIC \\
\hline LP & 1.58727 & 0.7910807 & -0.8855648 & 1.778844 \\
TP & 1.190114 & 0.7221261 & -0.1178869 & 2.546522 \\
\hline
\end{tabular}


Since it has the lowest AIC and BIC, the LP distribution is preferable to the TP distribution. Figure 8 displays the estimated pdfs of these two distributions.

Fits of Data set 2

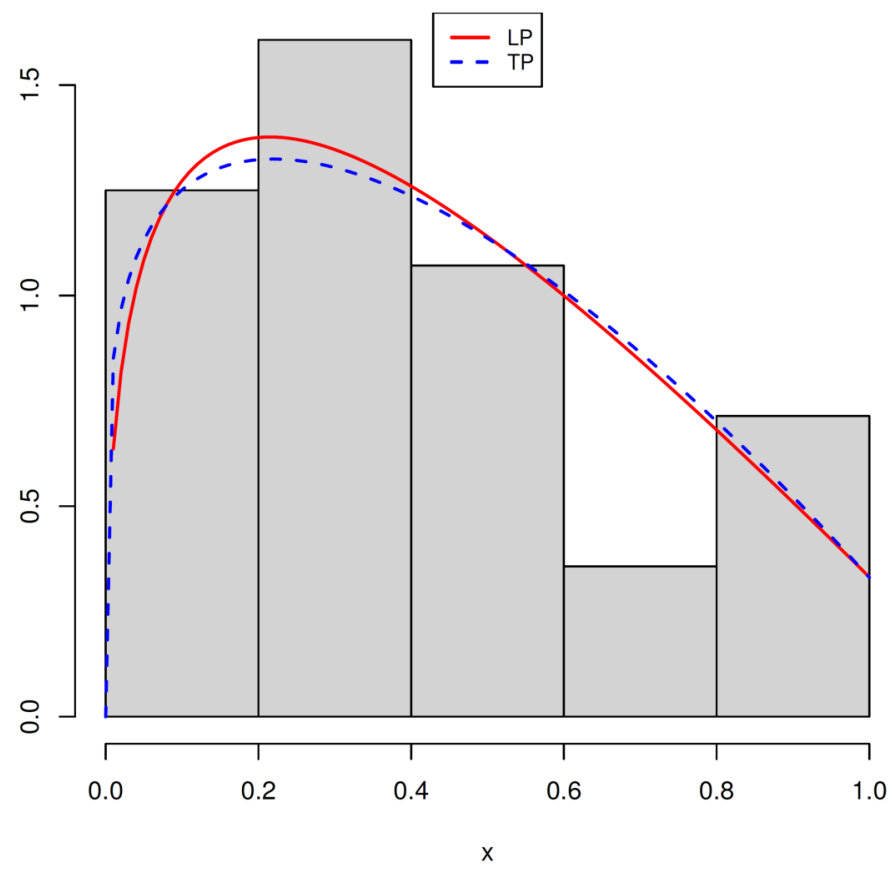

Figure 8. Curves of the estimated pdfs of the LP and TP distributions for Data set 2 .

Figure 8 shows that the difference between the two estimated pdfs is primarily for the values into $(0.2,0.4)$; the LP distribution is slightly better than the TP distribution on this interval.

- The third data set, called Data set 3, exhibits the vinyl chloride data collected from clean upgrading, monitoring wells in $\mathrm{mg} / \mathrm{L}$. It is derived from Bhaumik et al. [3]. The data set is: $\{5.1,1.2,1.3,0.6,0.5,2.4,0.5$, $1.1,8.0,0.8,0.4,0.6,0.9,0.4,2.0,0.5,5.3,3.2,2.7,2.9,2.5,2.3,1.0,0.2$, $0.1,0.1,1.8,0.9,2.0,4.0,6.8,1.2,0.4,0.2\}$. Again, we perform a "unit range" operation by dividing these data by 8.1 to obtain data ranging between $(0,1)$. 
Table 5 offers a simple statistical summary of the unit data set.

Table 5. Statistical description of Data set 3

\begin{tabular}{|l|l|}
\hline Mean & 0.2320261 \\
Median & 0.1419753 \\
St. deviation & 0.24106 \\
Variance & 0.05810995 \\
Skewness & 1.603688 \\
Kurtosis & 5.005408 \\
Minimum & 0.01234568 \\
Maximum & 0.9876543 \\
\hline
\end{tabular}

We remark that the data are right skewed, mesokurtic and with a wide range of values between $(0,1)$. In view of the shape properties of its pdf, the LP distribution is able to fit such data. The MLEs $\hat{\alpha}$ and $\hat{\lambda}$ of $\alpha$ and $\lambda$, respectively, and standard information criteria are presented in Table 6.

Table 6. Values of the MLEs, AIC and BIC for Data set 3

\begin{tabular}{|ccccc|}
\hline Distribution & MLE $\hat{\alpha}$ & MLE $\hat{\lambda}$ & AIC & BIC \\
\hline LP & 0.9404668 & 0.8811955 & -25.52001 & -22.46729 \\
TP & 0.6982504 & 0.8388123 & -24.38845 & -21.33572 \\
\hline
\end{tabular}

Since it has the lowest AIC and BIC, the LP distribution can be considered as the best from the fitting point of view. Figure 9 presents the estimated pdfs of these two distributions. 
Fits of Data set 3

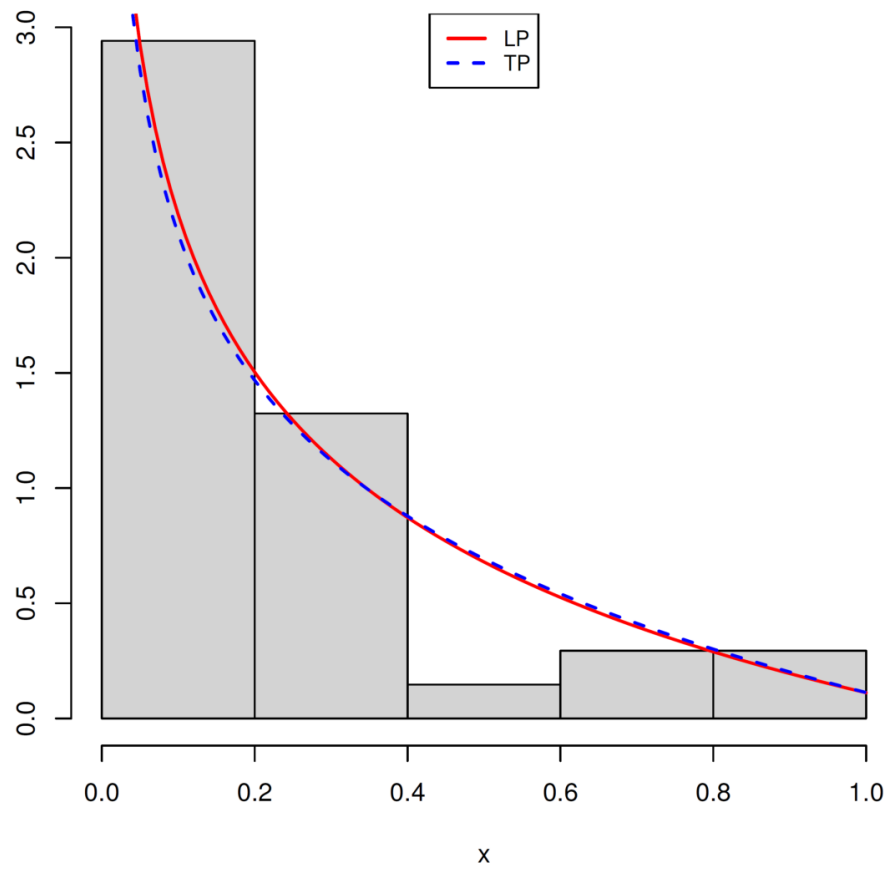

Figure 9. Curves of the estimated pdfs of the LP and TP distributions for Data set 3 .

The fits in Figure 9 are almost similar, showing that the LP distribution is also competitive for data well fitted by the TP distribution.

\section{Summary and Discussion}

In the last decade, researchers have been very interested in the idea of extending the power distribution. An interesting extension combining simplicity and applicability was given by the transmuted power (TP) distribution. In this article, we have proposed an alternative unit distribution, called the log-weighted power (LP) distribution. It is defined by modifying the cumulative distribution function of the TP distribution through the use of an original logarithmic weighted function. It can be seen as a simple variant of the log-Lindley distribution established by 
Gómez-Déniz et al. [16]. With its definition, a clear stochastic ordering implying power, transmuted power and log-weighted power distributions is established. Furthermore, some shape properties of the central functions of the LP distribution are not observed for the TP distribution, including a large panel of decreasing and sharp (mesokurtic) left skewed increasing-decreasing shapes for the probability density function, and flexible bathtub shapes for the hazard rate function. As a result, the LP distribution provides a statistical alternative to the TP distribution. With this motivation in mind, we have studied the LP distribution in-depth, exploring its generalized logarithmic moments, incomplete moments, Rényi entropy, order statistics, and reliability measures. We have also presented a panel of new distributions that naturally emerge from the LP distribution. The two parameters of the LP distribution are estimated using the maximum likelihood procedure for a data fitting objective. Then, using one artificial data set and two real data sets, we showed that the LP distribution can provide a better fit than the TP distribution.

Future directions of research include the investigation of univariate distributions derived from the LP distribution, the construction of bivariate distributions on the unit square with the LP distribution as the marginal distribution(s), the consideration of other estimation procedures, including those used in a more complex statistical setting with censored samples or others, and the development of regression-type models.

\section{Acknowledgement}

We would want to express our gratitude to the reviewers for their time and work in helping us improve our manuscript. 


\section{References}

[1] M. V. Aarset, How to identify bathtub hazard rate, IEEE Transactions Reliability 36(1) (1987), 106-108.

DOI: https://doi.org/10.1109/TR.1987.5222310

[2] Ahmed M. T. Abd El-Bar, M. do. C. S. Lima and M. Ahsanullah, Some inferences based on a mixture of power function and continuous logarithmic distribution, Journal of Taibah University for Science 14(1) (2020), 1116-1126.

DOI: https://doi.org/10.1080/16583655.2020.1804140

[3] D. K. Bhaumik, K. Kapur and R. D. Gibbons, Testing parameters of a gamma distribution for small samples, Technometrics 51(3) (2009), 326-334.

DOI: https://doi.org/10.1198/tech.2009.07038

[4] N. Balakrishnan and V. B. Nevzorov, A Primer on Statistical Distributions, John Wiley \& Sons, 2004.

[5] C. R. Brito, L. C. Régo, W. R. Oliveira and F. Gomes-Silva, Method for generating distributions and classes of probability distributions: The univariate case, Hacettepe Journal of Mathematics and Statistics 48(3) (2019), 897-930.

DOI: https://doi.org/10.15672/HJMS.2018.619

[6] G. Casella and R. L. Berger, Statistical Inference, Brooks/Cole Publishing Company: Bel Air, CA, USA, 1990.

[7] C. Chesneau, Study of a unit power-logarithmic distribution, Open Journal of Mathematical Sciences 5(1) (2021), 218-235.

DOI: https://doi.org/10.30538/oms2021.0159

[8] C. Chesneau, A note on an extreme left skewed unit distribution: Theory, modelling and data fitting, Open Statistics 2(1) (2021), 1-23.

DOI: https://doi.org/10.1515/stat-2020-0103

[9] C. Chesneau, L. Tomy and J. Gillariose, On a new distribution based on the arccosine function, Arabian Journal of Mathematics (2021), (to appear).

DOI: https://doi.org/10.1007/s40065-021-00337-x

[10] G. M. Cordeiro, R. B. Silva and A. D. C. Nascimento, Recent Advances in Lifetime and Reliability Models, Bentham Books, 2020.

DOI: https://doi.org/10.2174/97816810834521200101

[11] S. T. Dara and M. Ahmad, Recent Advances in Moment Distribution and their Hazard Rates, LAP Lambert Academic Publishing, GmbH, KG, 2012.

[12] M. Denuit, J. Dhaene, M. Goovaerts and R. Kaas, Actuarial Theory for Dependent Risks: Measures, Orders and Models, Jhon Wiley \& Sons, Ltd., 2006. 
[13] S. Ferrari and F. Cribari-Neto, Beta regression for modelling rates and proportions, Journal of Applied Statistics 31(7) (2004), 799-815.

DOI: https://doi.org/10.1080/0266476042000214501

[14] W. Gilchrist, Statistical Modelling with Quantile Functions, CRC Press, Abingdon, 2000 .

DOI: https://doi.org/10.1201/9781420035919

[15] R. E. Glaser, Bathtub and related failure rate characterizations, Journal of the American Statistical Association 75(371) (1980), 667-672.

DOI: https://doi.org/10.2307/2287666

[16] E. Gómez-Déniz, M. A. Sordo and E. Calderín-Ojeda, The log-Lindley distribution as an alternative to the beta regression model with applications in insurance, Insurance: Mathematics and Economics 54 (2014), 49-57.

DOI: https://doi.org/10.1016/j.insmatheco.2013.10.017

[17] I. S. Gradshteyn and I. M. Ryzhik, Table of Integrals, Series, and Products, Seventh Edition, Editors A. Jeffrey \& D. Zwillinger, Academic Press, Burlington, MA, 2007.

DOI: https://doi.org/10.1016/C2013-0-10754-4

[18] J. R. M. Hosking, L-moments: Analysis and estimation of distributions using linear combinations of order statistics, Journal of the Royal Statistical Society, Series B: Methodological 52(1) (1990), 105-124.

DOI: https://doi.org/10.1111/j.2517-6161.1990.tb01775.x

[19] N. L. Johnson, S. Kotz and N. Balakrishnan, Continuous Univariate Distributions, Volume 1, 2 Edition, John Wiley \& Sons, New York, 1994.

[20] C. Kleiber and S. Kotz, Statistical Size Distributions in Economics and Actuarial Sciences, Volume 470, John Wiley \& Sons, 2003.

[21] J. P. Klein and M. L. Moeschberger, Survival Analysis: Techniques for Censored and Truncated Data; Springer: Berlin/Heidelberg, Germany, 2006.

[22] S. Kotz, Y. Lumelskii and M. Pensky, The Stress-Strength Model and its Generalizations: Theory and Applications, World Scientific: Singapore, 2003.

[23] P. Kumaraswamy, A generalized probability density function for double-bounded random processes, Journal of Hydrology 46(1-2) (1980), 79-88.

DOI: https://doi.org/10.1016/0022-1694(80)90036-0

[24] K. S. Lomax, Business failures: Another example of the analysis of failure data, Journal of the American Statistical Association 49(268) (1954), 847-852.

DOI: https://doi.org/10.2307/2281544

[25] R. M. Mandouh and M. A.-G. Mohamed, A log-weighted power function distribution and its statistical properties, Journal of Data Sciences 18(2) (2020), 257-278.

DOI: https://doi.org/10.6339/JDS.202004_18(2).0003 
[26] N. U. Nair and P. G. Sankaran, Quantile-based reliability analysis, Communications in Statistics: Theory and Methods 38(2) (2009), 222-232.

DOI; https://doi.org/10.1080/03610920802187430

[27] R Core Team, R: A Language and Environment for Statistical Computing, R Foundation for Statistical Computing, Vienna, Austria, 2014.

URL http://www.R-project.org/

[28] A. Rényi, On measures of entropy and information, In: Proceedings of the 4th Berkeley Symposium on Mathematical Statistics and Probability, University of California Press, Berkeley 1 (1961), 547- 561.

[29] J. M. Ruiz and J. Navarro, Characterizations based on conditional expectations of the doubled truncated distribution, Annals of the Institute of Statistical Mathematics 48(3) (1996), 563-572.

DOI: https://doi.org/10.1007/BF00050855

[30] M. Q. Shahbaz, M. Ahsanullah, S. Hanif Shahbaz and B. M. Al-Zahrani, Ordered Random Variables: Theory and Applications, Atlantis Press and Springer, France, 2016.

DOI: https://doi.org/10.2991/978-94-6239-225-0

[31] M. N. Shahzad and Z. Asghar, Transmuted power function distribution: A more flexible distribution, Journal of Statistics and Management Systems 19(4) (2016), 519-539.

DOI: https://doi.org/10.1080/09720510.2015.1048096

[32] W. T. Shaw and I. R. Buckley, The alchemy of probability distributions: beyond Gram-Charlier expansions, and a skew-kurtotic-normal distribution from a rank transmutation map, arXiv preprint arXiv:0901.0434, (2009).

[33] C. Tanis, On transmuted power function distribution: Characterization, risk measures, and estimation, Journal of New Theory 34 (2021), 72-81.

[34] C. W. Topp and F. C. Leone, A family of J-shaped frequency functions, Journal of the American Statistical Association 50(269) (1955), 209-219.

DOI: https://doi.org/10.2307/2281107

[35] J. R. van Dorp and S. Kotz, The standard two-sided power distribution and its properties: With applications in financial engineering, The American Statistician 56(2) (2002), 90-99.

[36] A. Zaka, A. S. Akhter and R. Jabeen, A view on characterizations of the J shaped statistical distribution, Indian Journal of Science and Technology 13(32) (2020), 3327-3338.

DOI: https://doi.org/10.17485/IJST/v13i32.353 